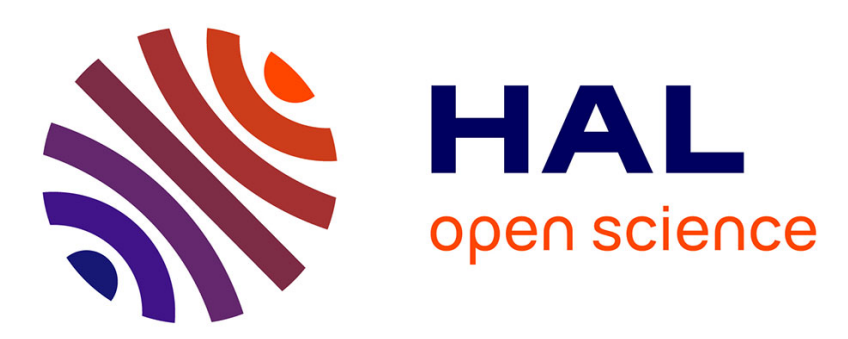

\title{
Uniqueness and Hölder stability of discontinuous diffusion coefficients in three related inverse problems for the heat equation \\ Olivier Poisson
}

\section{- To cite this version:}

Olivier Poisson. Uniqueness and Hölder stability of discontinuous diffusion coefficients in three related inverse problems for the heat equation. 2007. hal-01255486

\author{
HAL Id: hal-01255486 \\ https://hal.science/hal-01255486
}

Preprint submitted on 13 Jan 2016

HAL is a multi-disciplinary open access archive for the deposit and dissemination of scientific research documents, whether they are published or not. The documents may come from teaching and research institutions in France or abroad, or from public or private research centers.
L'archive ouverte pluridisciplinaire HAL, est destinée au dépôt et à la diffusion de documents scientifiques de niveau recherche, publiés ou non, émanant des établissements d'enseignement et de recherche français ou étrangers, des laboratoires publics ou privés. 


\title{
Uniqueness and Hölder stability of discontinuous diffusion coefficients in three related inverse problems for the heat equation
}

\author{
Olivier Poisson \\ Laboratoire d'analyse, topologie, probabilités, CNRS UMR 6632 \\ Université de Provence, Marseille, France \\ E-mail: poisson@latp.univ-mrs.fr
}

\begin{abstract}
We consider the heat equation $\partial_{t} y-\operatorname{div}(c \nabla y)=H$ with a discontinuous coefficient in three connected situations. We give uniqueness and stability results for the diffusion coefficient $c(\cdot)$ in the main case from measurements of the solution on an arbitrary part of the boundary and at a fixed time in the whole spatial domain. The diffusion coefficient is assumed to be discontinuous across an unknown interface. The key ingredients are a Carleman-type estimate with non-smooth data near the interface and a stability result for the discontinuous coefficient $c(\cdot)$ in an inverse problem associated with the stationary equation $-\operatorname{div}(c \nabla u)=f$.
\end{abstract}

AMS classification scheme numbers: 35K05,35R30

\section{Introduction}

\subsection{Equations with discontinuous diffusion coefficient}

This article is devoted to the question of the identification of a diffusion coefficient, $c$, for a heat transfer problem in a bounded domain, with the main particularity that $c$ is discontinuous. Such regularity can be encountered in the case of embedded materials.

Let $\Omega \subset \mathbb{R}^{n}$ be a bounded connected open set. The set $\bar{\Omega}$ is assumed to be a $\mathcal{C}^{2}$ submanifold with boundary $\partial \Omega=\Gamma$ in $\mathbb{R}^{n}$ (see e.g. [1, Definition 1.2.1.2]).

Let $T>0$ and let $\mathcal{L}:=\partial_{t}-\operatorname{div}(c \nabla \cdot)$ be the formal heat operator in the cylinder $Q_{0}:=(0, T) \times \Omega$. We consider the following problem for the heat equation:

$$
\left\{\begin{array}{l}
\mathcal{L} y=0 \text { in } \mathcal{D}^{\prime}\left(Q_{0}\right), \\
y(0, x)=y_{0}(x) \text { in } \Omega, \\
y(t, x)=h(t, x) \text { on } \Sigma_{0}:=(0, T) \times \Gamma .
\end{array}\right.
$$

The diffusion coefficient $c$ shall be kept independent of time $t$. It is a measurable function in $\Omega$. The boundary condition $h(t, x)$ on $\Sigma_{0}=[0, T] \times \Gamma$ is assumed sufficiently smooth and shall be kept fixed. For $y_{0} \in L^{2}(\Omega)$ and $h$ sufficiently smooth on $\Sigma_{0}$, if $c$ satisfies $0<c_{\min } \leq c(x) \leq c_{\max }<\infty$, then we can prove (e.g., [2]) that problem (1.1) admits 
an unique solution $y \in \mathcal{C}^{0}\left([0, T] ; L^{2}(\Omega)\right)$. We denote it by $Y\left(h, y_{0}\right)$.

We assume that diffusion coefficients belong to the set $\mathcal{E} \subset L^{\infty}(\Omega)$ described by the following conditions (i),(ii),(iii):

(i) $0<c_{\min } \leq c(\cdot) \leq c_{\max }<\infty$;

(ii) $c(\cdot)$ is piecewise smooth in $\Omega$, that is: $\Omega$ admits a partition in two non empty open sets $\Omega_{0}=\Omega \backslash \overline{\Omega_{1}}, \Omega_{1} \subset \subset \Omega$ which are separated by an interface: $S=\overline{\Omega_{0}} \cap \overline{\Omega_{1}}=$ $\partial \Omega_{1} \subset \Omega$ is at least of class $\mathcal{C}^{2}$; we have $c_{\mid \Omega_{j}}=: c_{j}$ and $c_{j} \in \mathcal{C}^{2}\left(\bar{\Omega}_{j}\right)$ is arbitrarily extended in $\mathcal{C}^{2}(\bar{\Omega}), j=0,1$;

(iii) $c_{0}(x) \leq c_{1}(x), \forall x \in S$.

\subsection{Statement of the main inverse problem}

Let $t_{0} \in(0, T)$ and $T^{\prime} \in(0, T)$ be arbitrarily fixed and $\gamma \neq \emptyset$ be an arbitrary relatively open subset of $\Gamma$ (see figure 1). We denote $\gamma_{T}:=\left(t_{0}, T\right) \times \gamma$. In problem (1.1), we fix the boundary condition $y(t, x)=h(t, x)=0$ for $0 \leq t \leq t_{0}, x \in \Gamma$.

If we change the diffusion coefficient $c$ into $c^{\prime}$ we denote by $y^{\prime}=Y^{\prime}\left(h, y_{0}^{\prime}\right)$ the solution of (1.1) associated to $c^{\prime}$ and $y_{0}^{\prime}$ for initial condition.

The interface depends on the diffusion coefficient and is an unknown of the following inverse problem.

Problem: to select $l \in \mathbb{N}^{*}, h_{j} \in \mathcal{C}^{0}(\Sigma), 1 \leq j \leq l$ suitably and to determine the diffusion coefficient $c$ with the interface $S$ by observation data $c_{\mid \gamma},\left(\partial_{n} y_{j}\right)_{\mid \gamma_{T}}, y_{j}\left(T^{\prime}, \cdot\right)_{\mid \Omega}$, $1 \leq j \leq l$, where $y_{j}=Y\left(h_{j}, y_{0}\right)$.

Note that in the formulation of the inverse problem, the initial values are also unknown. The nonhomogeneous terms $h_{j}$ are considered as inputs to equation (1.1) and $\left(\partial_{n} y_{j}\right)_{\mid \gamma_{T}}, y_{j}\left(T^{\prime}, \cdot\right)_{\mid \Omega}$ are outputs.

Figure 1. Case 1

\subsection{Main result}

The main result is local uniqueness and stability for $c$ with only two measurements. This result requires the following additional assumptions. Setting $n^{\prime}:=[n / 4]+1, \Gamma$ is of class $\mathcal{C}^{2 n^{\prime}+2}$; the set $\mathcal{E}$ of diffusion coefficients is restricted by the additional condition (iv): $S$ is of class $\mathcal{C}^{2 n^{\prime}+2}$ and $c_{\mid \Omega_{j}} \in \mathcal{C}^{2 n^{\prime}+1}\left(\overline{\Omega_{j}}\right), j=0,1$.

Local Uniqueness: Let $c \in \mathcal{E}$. For any initial data $y_{0}$ there exist two boundary conditions $h_{i}(t, x), i=1,2$, on $\Sigma$ such if $c^{\prime} \in \mathcal{E}$ with $c^{\prime}=c$ on $\gamma, y_{i}^{\prime}\left(T^{\prime}\right)=y_{i}\left(T^{\prime}\right)$ in $\Omega$ and $\left(\partial_{n} y_{i}^{\prime}\right)_{\left.\right|_{\gamma_{T}}}=\left(\partial_{n} y_{i}\right)_{\left.\right|_{\gamma_{T}}}$ in $\gamma_{T}, i=1,2$, then $c^{\prime}=c$ in $\Omega$.

Here the notation is $y_{i}=Y\left(h_{i}, y_{0}\right)$ and $y_{i}^{\prime}=Y^{\prime}\left(h_{i}, y_{0}\right)$.

Stability: Moreover we have the following estimate

$\left\|c-c^{\prime}\right\|_{L^{2}(\Omega)} \leq C\left(\left\|c-c^{\prime}\right\|_{L^{2}(\gamma)}+\sum_{i=1,2}\left\|\partial_{n}\left(y_{i}-y_{i}^{\prime}\right)\right\|_{L^{2}\left(\gamma_{T}\right)}+\left\|\partial_{t} \partial_{n}\left(y_{i}-y_{i}^{\prime}\right)\right\|_{L^{2}\left(\gamma_{T}\right)}\right.$ 


$$
\left.+\left\|\nabla\left(y_{i}\left(T^{\prime}\right)-y_{i}^{\prime}\left(T^{\prime}\right)\right)\right\|_{L^{\infty}(\Omega)}^{\frac{1}{2}}\right) .
$$

The constant $C$ in (1.2) depends on $c, c^{\prime}, h_{i}, i=1,2$. Let us precise right here how we choose $h_{i}, i=1,2$. They vanishe outside $\gamma_{T}: h_{i}(t, x)=0$ if $x \in \Gamma \backslash \gamma$; they are sufficiently smooth (as smooth as $\gamma$ ). They ensure a condition of the following kind: (H1a): $\sum_{i=1,2}\left|\nabla y_{i}\left(T^{\prime}, \cdot\right) \cdot \nabla \beta^{(i)}\right| \geq \delta>0$ in $\Omega \backslash \omega$, where functions $\beta^{(i)}, i=1,2$ depend only on the geometry of $(S, \Omega, \gamma)$ and on the values of $c_{0} / c_{1}$ on $S$. Their full properties are described below (see section 2 ).

\subsection{Comparison with existing results}

The key ingredients to this stability result are global Carleman estimates for the operator $\mathcal{L}$ and its formal adjoint $\mathcal{L}^{*}:=-\partial_{t}-\operatorname{div}(c \nabla \cdot)$ on the cylinder $Q$, and a stability result for some related stationary inverse problem.

To achieve uniqueness and stability results, the use of global Carleman estimates was first introduced in [3, 4], then in [5] and then by others, e.g. [6]. For literature on Carleman estimates we refer to [7] for local estimates on the parabolic case. For global estimates with data in $L^{2}(Q)$ or in $H^{-1}(Q)$ we refer to $[8,9]$.

Stability results for Parabolic equations are recent, to my knowledge (see [4, 10], [11, Chapters 3,4$]$ ). For uniqueness and stability results for the similar hyperbolic inverse problem, we refer to $[12,13]$ where the coefficient $c$ is smooth and is involved in the divergence form.

Apart from [5] where Lipschitz stability estimates are obtained with the condition that coefficients are smooth, there are few results on Lipschitz stability, even for linear cases; see $[14,15]$ for parabolic equations and $[16]$ for the hyperbolic case.

The main difficulty in the present problem is to deal with discontinuous diffusion coefficients. As opposed to [15], the interface is not fixed; in addition, authors of [15] assumed $c-c^{\prime}$ to be constant in each subdomain $\Omega_{j}, j=0,1$. We shall not make such a restriction here. The controllability of such parabolic equations was studied in [17]. Null-controllability is achieved via an observability inequality for the adjoint system, which is deduced in [17] from a global Carleman estimate yet assuming a monotonicity on the coefficient $c$ in connection to the observation location. As authors of [15] I underline that to achieve a stability result we have to derive some Carleman estimate for the difference of the two solutions, $y_{i}, y_{i}^{\prime}$, which includes additional interface terms. From there our method differs from that of [15]. As opposed to [15], we do not look for estimates on $\nabla\left(y_{i}-y_{i}^{\prime}\right)$ in $L^{2}(Q)$, but only on $y_{i}-y_{i}^{\prime}$. Since the Carleman estimates in [17] are only proved for sufficiently smooth functions in $\left[t_{0}, T\right] \times \overline{\Omega_{j}}, j=0,1$, that have to satisfy two transmission conditions on $S$, we show at first that their results can be extended to a larger class of functions; then by using a technique as in [18], we are able to derive our useful Carleman estimates for functions that do not satisfy this transmission conditions.

This point of view has many advantages. Firstly, we do not require $c_{1}-c_{0} \geq \Delta>0$ on $S$ which is assumed in [15, Theorem 2.2]. Secondly, our Carleman estimate with 
data in $H^{-1}(Q)$ is straightforwardly obtained by the general method developed in [9], and calculations are thus significantly reduced. Thirdly, the fact that the coefficient $c$ is discontinuous has no importance in our method and consequently, as opposed to [15], there is no integral on $S$ among the dominating terms. This is the reason why $c-c$ is not forced to be piecewise constant.

To solve inverse problems cats new light on a great difference between operators $\partial_{t}-\operatorname{div}(c \nabla \cdot)$ and $\partial_{t}-c \Delta \cdot\left(\right.$ or $\left.\partial_{t}-\Delta \cdot+V\right)$. In the second one, the knowledge of a function $u$ and $c \Delta u$ provides algebrically $c=(c \Delta u) /(\Delta u)$. Obviously we do not have such an easy relation with the first one. In fact, the same difficulties arise in the study of the stationary equations. This is why to set and to solve an adequate inverse problem for the equation $-\operatorname{div}(c \nabla u)=f$ are crucial. If $c$ is piecewise constant then $-\operatorname{div}(c \nabla u)$ operates in $\Omega \backslash S$ as $-c \Delta u$ and so the knowledge of $u$ and $\operatorname{div}(c \nabla u)$ in $\Omega$ provides algebrically $c=(\operatorname{div}(c \nabla u)) /(\Delta u)$ again. In equation $-\operatorname{div}(c \nabla u)=f$, we consider that the unknown is $c(x)$ and the data are $f, u$. So we treat a first-order partial differential equation in which the unknown function is not restricted to be piecewise constant. The uniqueness question (for $c$ ) could be analysed in a more general framework (for example without additional smoothness on $u$ near $S$ ) but this would go beyond the scope of this article. In fact we need weighted $L^{2}$-estimate, with the Carleman functions described in section 2 at some fixed time $T^{\prime}$ as weights. An estimate of this sort can be found in [19, Lemma 3.2] in the case of smooth coefficient: it is obtained by comparison between leading terms of a scalar product performed in the Hilbert space $L^{2}(\Omega, \mathrm{d} x)$. Here the non smoothness of $c$ yields two difficulties. Firstly, integration by parts requires the De Giorgi-Federer formula (see for e.g. [20, section 5.8]) instead of the Green's one. To my knowledge, this technique was not essential until now to solve such problems. Secondly, integrals on $S$ and $S^{\prime}$ appear and have to be expressed in term of $u-u^{\prime}$ (where $S^{\prime}$ denotes the interface for a diffusion coefficient $c^{\prime}$ which is solution of the stationary equation with data $\left.f^{\prime}, u^{\prime}\right)$. This can not be done with scalar products in $L^{2}(\Omega, \mathrm{d} x)$ and is the reason why scalar products are made in the space $L^{2}(\Omega, c(x) \mathrm{d} x)$.

The result of this difficulty is that the estimate (1.2) is not Lipschitz (du to the power $\frac{1}{2}$ for the term $\left.\left\|\nabla\left(y_{i}\left(T^{\prime}\right)-y_{i}^{\prime}\left(T^{\prime}\right)\right)\right\|_{L^{\infty}(\Omega)}\right)$ : this originates from integrals on $\left(S \backslash S^{\prime}\right) \cup\left(S^{\prime} \backslash S\right)$.

Let us resume the method and the ideas of dealing with the discontinuity. The method is composed of three parts. Firstly, we obtain a Carleman estimate for very general functions that do not necessarily satisfy transmission conditions, as functions with the factor term $\left(c-c^{\prime}\right)$. Secondly we treat a somewhat inverse problem for a stationary equation with a discontinuous coefficient equation as a first-order partial differential equation where the unknown belongs to a set of a discontinuous functions.

Notice that these two parts are independent to each other. Furthermore they may have interest outside inverse problems for parabolic equations. Thirdly, we use both ingredients above in the method of Bukhgeim and Klibanov. 


\subsection{Two other connected problems}

Some technical difficulties are due to the place where data are observed - on $\gamma$ - and can be reduced if instead of this geometrical situation which is refered to as "case 1" we consider the two following ones refered to as "case 2" and "case 3" (see figure 2). We shall treat successively case 3, case 2 and case 1 since this represents an increasing order of difficulty. Indeed, the results for case 2 are obtained from computations for case 3 by slight modifications on the weight functions appearing in the Carleman estimates, and the case 1 can almost be seen as case 2 if the domain $\Omega$ is extended.

Figure 2. Cases 2,3

Case 2 - We consider measurements in an open set $\omega \subset \subset \Omega_{0}$. Equations (1.1) are replaced by

$$
\left\{\begin{array}{l}
\mathcal{L} y=H \chi_{\omega} \text { in } Q_{0} \\
y(0, x)=y_{0}(x) \text { in } \Omega \\
y(t, x)=0 \text { on } \Sigma_{0}
\end{array}\right.
$$

where $H(t, x)$ denotes source terms. We fix $H(t, x)=0$ for $t \in\left[0, t_{0}\right]$. If $H \in L^{2}(Q)$ then there exists an unique solution $y \in L^{2}\left(0, T ; H_{0}^{1}(\Omega)\right)$ of $(1.3)$. We denote it by $Y\left(H, y_{0}\right)$. Besides $Y\left(H, y_{0}\right) \in \mathcal{C}^{0}\left([0, T] ; H_{0}^{1}(\Omega)\right)$. Furthermore if $H$ is sufficiently smooth (for example $H \in \mathcal{C}_{0}^{\infty}([0, T] \times \omega)$, then $Y\left(H, y_{0}\right) \in \mathcal{C}^{r}\left(\left[t_{0}, T\right] ; D\left(A^{k}\right)\right.$ ) for all $r, k \in \mathbb{N}$, where $A$ denotes the following spatial self adjoint operator in $L^{2}(\Omega ; \mathrm{d} x)$ :

$$
A u:=-\operatorname{div}(c \nabla u), D(A):=\left\{u \in H_{0}^{1}(\Omega), A u \in L^{2}(\Omega)\right\} .
$$

To set the inverse problem we fix inputs $H_{i}, i=1, \cdots, l$ in $\omega_{T}=\left(t_{0}, T\right) \times \omega$ and we measure the function $c(\cdot)$ in $\omega, y_{i}(t, \cdot)$ in $\omega_{T}, y_{i}\left(T^{\prime}, \cdot\right)$ in $\Omega$ at a time $T^{\prime} \in\left(t_{0}, T\right)$, where $y_{i}=Y\left(H_{i}, y_{0}\right)$.

In case 2 , we prove the following result for uniqueness and stability with only two measurements.

We assume that $\Gamma$ is of class $\mathcal{C}^{2 n^{\prime}+2}$ and that the set $\mathcal{E}$ is restricted by the additional condition (iv).

Local Uniqueness: Let $c \in \mathcal{E}$. For any initial data $y_{0}$ there exist two source terms $H_{i}(t, x), i=1,2$, on $\omega_{T}$ such that if $c^{\prime} \in \mathcal{E}$ with $c^{\prime}=c$ in $\omega, y_{i}^{\prime}\left(T^{\prime}\right)=y_{i}\left(T^{\prime}\right)$ in $\Omega$ and $y_{i}^{\prime}=y_{i}$ in $\omega_{T}, i=1,2$, then $c^{\prime}=c$ in $\Omega$.

Here $y_{i}^{\prime}(t, x)=Y^{\prime}\left(H_{i}, y_{0}^{\prime}\right)$ denotes the solution of $(1.3)$ with $\left(c, y_{0}\right)$ replaced by $\left(c^{\prime}, y_{0}^{\prime}\right)$ and $H=H_{i}$.

Stability: Moreover we have the following estimate

$$
\left\|c-c^{\prime}\right\|_{L^{2}(\Omega)} \leq C\left(\left\|c-c^{\prime}\right\|_{L^{2}(\omega)}+\sum_{i=1,2}\left\|\partial_{t}\left(y_{i}-y_{i}^{\prime}\right)\right\|_{L^{2}\left(\omega_{T}\right)}\right.
$$


Uniqueness of discontinuous diffusion coefficients for the heat equation

$$
\left.+\left\|\partial_{t t}^{2}\left(y_{i}-y_{i}^{\prime}\right)\right\|_{L^{2}\left(\omega_{T}\right)}+\left\|\nabla\left(y_{i}\left(T^{\prime}\right)-y_{i}^{\prime}\left(T^{\prime}\right)\right)\right\|_{L^{2}(\Omega)}^{\frac{1}{2}}\right) .
$$

The constant $C$ in (1.4) depends on $c, c^{\prime}, H_{i}, i=1,2$.

Case 3 - It is similar to case 2 , but places of $\Omega_{0}$ and $\Omega_{1}$ are inverted. In case 3 , we shall prove the following result for uniqueness and stability with only one measurement: assume that $\Gamma$ is of class $\mathcal{C}^{2 n^{\prime}+2}$ and that the set $\mathcal{E}$ is restricted by the additional condition (iv);

Local Uniqueness: Let $c \in \mathcal{E}$. For any initial data $y_{0}$ there exists a source term $H(t, x)$ on $\omega_{T}$ such that if $c^{\prime} \in \mathcal{E}$ with $c^{\prime}=c$ in $\omega, y^{\prime}\left(T^{\prime}\right)=y\left(T^{\prime}\right)$ in $\Omega$ and $y^{\prime}=y$ in $\omega_{T}$, then $c^{\prime}=c$ in $\Omega$.

Here $y=Y\left(H, y_{0}\right)$ and $y^{\prime}(t, x)=Y^{\prime}\left(H, y_{0}^{\prime}\right)$.

Stability: Moreover we have the following estimate

$$
\begin{aligned}
\left\|c-c^{\prime}\right\|_{L^{2}(\Omega)} \leq & C\left(\left\|c-c^{\prime}\right\|_{L^{2}(\omega)}+\left\|\partial_{t}\left(y-y^{\prime}\right)\right\|_{L^{2}\left(\omega_{T}\right)}+\right. \\
& \left.\left\|\partial_{t t}^{2}\left(y-y^{\prime}\right)\right\|_{L^{2}\left(\omega_{T}\right)}+\left\|\nabla\left(y\left(T^{\prime}\right)-y^{\prime}\left(T^{\prime}\right)\right)\right\|_{L^{2}(\Omega)}^{\frac{1}{2}}\right) .
\end{aligned}
$$

The constant $C$ in (1.5) depends on $c, c^{\prime}, H$. This paper is organized as follows. In Section 2 we derive Carleman estimates for the three considered cases. In Section 3 we prove the main estimate (1.5) for $c-c^{\prime}$ for case 3; in Subsection 3.1 we treat the related stationary inverse problem and we give the main estimate for $c-c^{\prime}$ (see (3.1)); in Subsection 3.2 we treat the non stationary inverse problem and we conclude for (1.5) under some assumptions. In Subsection 3.3 we show that these assumptions can be achieved. In Section 4 we derive from Section 3 the main requirement (1.4) for $c-c^{\prime}$ for case 2. In Section 5 we derive from Section 4 the main requirement (1.2) for $c-c^{\prime}$ for case 1 .

\section{Carleman estimates}

\subsection{Geometry and weights}

We first introduce some notation. In all cases, the outward unit normal on $\Gamma$ (respectively $\left.\partial \Omega_{j}, j=0,1\right)$ is denoted by $n$ (respectively $n_{j}$ ). The unit normal on $S$ denoted by $n_{S}$ or simply by $n=n_{1}=-n_{0}$ points towards $\Omega_{0}$. Formally, the jump of a quantity $q$ on $S$ is defined by $[q]:=\left(q \mid \Omega_{1}\right)_{\mid S}-\left(q \mid \Omega_{0}\right)_{\mid S}$.

For geometrical assumptions on the open subsets $\Omega_{j}, j=0,1$, and the construction of the weight functions we refer to [17]. The geometrical assumptions are fulfilled for usual domains, see for example the cases of figure 3 .

Figure 3. The Geometric assumptions are fulfilled in situations (a,c,e) for case 3, in $(\mathrm{f}, \mathrm{h}, \mathrm{i})$ for case 2 and in $(\mathrm{g})$ for case 1 . It is not fulfilled in situations (b,d) for case 3 and in (j) for case 3 . 
Case 3- (see [17, lemma 3.1]). There exists a function $\beta(x)$ such that:

- $\beta \in \mathcal{C}^{0}(\bar{\Omega}), \beta_{\mid \Omega_{j}} \in \mathcal{C}^{2}\left(\overline{\Omega_{j}}\right), \operatorname{div}(c \nabla \beta) \in L^{2}(\Omega)$.

- $\beta(x)>0$ for all $x \in \bar{\Omega} ; \beta_{\mid S}$ and $\beta_{\mid \Gamma}$ are constant.

- $\partial_{n} \beta(x)<0$ for all $x \in \Gamma$.

- $\left[c \partial_{n} \beta\right]=0$ and $\left(c \partial_{n} \beta\right)(x)>0$ for all $x \in S$.

- $\nabla\left(\beta_{\mid \Omega_{j}}\right)(x) \neq 0$ for all $x \in \overline{\Omega_{j}} \backslash \omega, j=0,1$.

- $\max _{\bar{\Omega}} \beta \leq 2 \min _{\bar{\Omega}} \beta$.

We introduce now Carleman weight functions. They have the form $s^{a} \lambda^{b} \varphi^{a} \mathrm{e}^{-d s \eta}$, with $a, b, d$ as numerical parameters, the parameters $s, \lambda$ taken sufficiently large and where we set:

$$
\varphi(t, x):=\frac{\mathrm{e}^{\lambda \beta(x)}}{\left(t-t_{0}\right)(T-t)} \text { and } \eta(t, x):=\frac{\mathrm{e}^{\lambda \bar{\beta}}-\mathrm{e}^{\lambda \beta(x)}}{\left(t-t_{0}\right)(T-t)},
$$

and $\bar{\beta}:=\frac{5}{4} \max _{\bar{\Omega}} \beta$. Note that these functions satisfy the following properties:

- $\nabla \varphi=\lambda \varphi \nabla \beta$;

- $\nabla \eta=-\lambda \varphi \nabla \beta$;

- (see [17, equation (90)])

$$
\left|\partial_{t} \eta\right|+\left|\partial_{t} \varphi\right| \leq C\left(T, t_{0}\right) \varphi^{2}
$$

Case 2- (see [17, lemma 3.2 and page 639]). We have $\Omega_{0} \subset \subset \Omega$ and $\omega \subset \subset \Omega_{1}$. Let $\omega_{i} \subset \subset \omega_{i}^{\prime} \subset \subset \Omega_{0}, i=1,2$, be four non empty open sets with $\mathrm{d}\left(\omega_{1}^{\prime}, \omega_{2}^{\prime}\right)>0$. There exist two functions $\beta^{(i)}, i=1,2$ such that:

- $\beta^{(i)} \in \mathcal{C}^{0}(\bar{\Omega}), \beta^{(i)}{ }_{\mid \Omega_{j}} \in \mathcal{C}^{2}\left(\overline{\Omega_{j}}\right), \operatorname{div}\left(c \nabla \beta^{(i)}\right) \in L^{2}(\Omega)$.

- $\beta^{(i)}(x)>0$ for all $x \in \bar{\Omega}, \beta^{(i)}{ }_{\mid S}$ and $\beta^{(i)}{ }_{\mid \Gamma}$ are constant.

- $\partial_{n} \beta^{(i)}(x)<0$ for all $x \in \Gamma$.

- $\left[c \partial_{n} \beta^{(i)}\right]=0$ and $c \partial_{n} \beta^{(i)}(x)>0$ for all $x \in S$.

- $\beta^{(2)} \geq 2 \beta^{(1)}$ in $\omega_{1}^{\prime}$ and $\beta^{(1)} \geq 2 \beta^{(2)}$ in $\omega_{2}^{\prime}$.

- $\nabla\left(\beta_{\mid \Omega_{j}}^{(i)}\right)(x) \neq 0$ for all $x \in \overline{\Omega_{j}} \backslash\left(\omega \cup \omega_{i}\right), i=1,2, j=0,1$.

- $\max _{k=1,2} \max _{\bar{\Omega}} \beta^{(k)} \leq 2 \beta^{(i)}(x), i=1,2, x \in \Omega$.

The weight functions have the form $\sum_{j=1,2} s^{a} \lambda^{b} \varphi_{j}^{a} \mathrm{e}^{-d s \eta_{j}}$ where we set

$$
\varphi_{j}(t, x):=\frac{\mathrm{e}^{\lambda \beta^{(j)}(x)}}{\left(t-t_{0}\right)(T-t)} \text { and } \eta_{j}(t, x):=\frac{\mathrm{e}^{\lambda \bar{\beta}}-\mathrm{e}^{\lambda \beta^{(j)}(x)}}{\left(t-t_{0}\right)(T-t)},
$$

and $\bar{\beta}:=\frac{5}{4} \max _{j=1,2} \max _{\bar{\Omega}} \beta^{(j)}$. Note that these functions satisfy the following properties:

- $\nabla \varphi_{j}=\lambda \varphi_{j} \nabla \beta^{(j)}$;

- $\nabla \eta_{j}=-\lambda \varphi_{j} \nabla \beta^{(j)}$

- $\left|\partial_{t} \eta_{j}\right|+\left|\partial_{t} \varphi_{j}\right| \leq C\left(T, t_{0}\right) \varphi_{j}^{2}$. 
Case 1- (see [15]). Let $\omega_{i} \subset \subset \omega_{i}^{\prime} \subset \subset \Omega_{1}, i=1,2$, be four non empty open sets with $\mathrm{d}\left(\omega_{1}^{\prime}, \omega_{2}^{\prime}\right)>0$. There exist two functions $\beta^{(i)}(x), i=1,2$ such that:

- $\beta^{(i)} \in \mathcal{C}^{0}(\bar{\Omega}), \beta^{(i)}{ }_{\mid \Omega_{j}} \in \mathcal{C}^{2}\left(\overline{\Omega_{j}}\right), \operatorname{div}\left(c \nabla \beta^{(i)}\right) \in L^{2}(\Omega)$.

- $\beta^{(i)}(x)>0$ for all $x \in \bar{\Omega}, \beta_{\mid S}^{(i)}$ and $\beta_{\mid \Gamma \backslash \gamma}^{(i)}$ are constant.

- $\partial_{n} \beta^{(i)}(x)<0$ for all $x \in \Gamma \backslash \gamma$.

- $\left[c \partial_{n} \beta^{(i)}\right]=0$ and $\left(c \partial_{n} \beta^{(i)}\right)(x)>0$ for all $x \in S$.

- $\beta^{(2)} \geq 2 \beta^{(1)}$ in $\omega_{1}^{\prime}$ and $\beta^{(1)} \geq 2 \beta^{(2)}$ in $\omega_{2}^{\prime}$.

- $\nabla \beta_{\mid \Omega_{j}}^{(i)}(x) \neq 0$ for all $x \in \overline{\Omega_{j}} \backslash \omega_{i}, i=1,2, j=0,1$.

- $\max _{k=1,2} \max _{\bar{\Omega}} \beta^{(k)} \leq 2 \beta^{(i)}(x), i=1,2, x \in \Omega$.

The construction of the functions $\beta^{(i)}$ is explained for instance in [15]: we consider a geometrical domain $\hat{\Omega}$ that extends $\Omega$ near a part $\gamma^{\prime} \subset \subset \gamma$ of the boundary (see figure 3). Let $\omega$ be an non empty open set with $\omega \subset \subset \hat{\Omega} \backslash \Omega$. We can construct

now geometrical functions $\beta^{(i)}$ associated with the couple $(\hat{\Omega}, \omega)$ as in case 2 . Then we consider restrictions of $\beta^{(i)}$ to the domain $\Omega$. By the same procedure we construct the weight functions.

The weight functions $\varphi_{j}, \eta_{j}$ are defined by (2.2) with the above modification of $\beta^{(j)}$.

Figure 4. Extension of $\Omega$

\subsection{Carleman estimates}

This is the first key ingredient for solving our inverse problem.

We recall that the heat operator is given by: $\mathcal{L} q=\partial_{t} q-\operatorname{div}(c \nabla q)$ and its formal adjoint is given by $\mathcal{L}^{*} q=-\partial_{t} q-\operatorname{div}(c \nabla q)$.

Let $A:=-\operatorname{div}(c \nabla \cdot)$ be the spatial self adjoint operator in $L^{2}(\Omega ; \mathrm{d} x)$ with domain $D(A):=\left\{u \in H_{0}^{1}(\Omega), A u \in L^{2}(\Omega)\right\}$. We set

$$
\begin{aligned}
& D(L):=\left\{q: q \in L^{2}\left(t_{0}, T ; H_{0}^{1}(\Omega)\right), \mathcal{L} q \in L^{2}(Q)\right\} \\
& D\left(L^{*}\right):=\left\{q: q \in L^{2}\left(t_{0}, T ; H_{0}^{1}(\Omega)\right), \mathcal{L}^{*} q \in L^{2}(Q)\right\} .
\end{aligned}
$$

Remark 2.1 Notice that if $q \in D(L)$ then $q_{\mid \Sigma}=0$ and $A q \in L^{2}\left(t_{0}, T ; H^{-1}(\Omega)\right)$. Hence $\partial_{t} q \in L^{2}\left(t_{0}, T ; H^{-1}(\Omega)\right)$ and $q\left(t_{0}\right)$ makes sense: $q\left(t_{0}\right) \in L^{2}\left(t_{0}, T ; H^{-1}(\Omega)\right)$.

We denote by $L$ (respectively $L^{*}$ ) the operator $\mathcal{L}$ (respectively $\mathcal{L}^{*}$ ) with domain $D(L)$ (respectively $D\left(L^{*}\right)$ ).

We assume in all cases that $q \in L^{2}(Q)$. The following estimates hold for $\lambda>\lambda_{0}$, $s>s_{0}(\lambda)$ for some $\lambda_{0}>0, s_{0}(\lambda)>0$. 
Case 3-

- If $q \in D\left(L^{(*)}\right)$ then we have

$$
\begin{aligned}
& s \lambda^{2} \int_{Q} \varphi \mathrm{e}^{-2 s \eta}|\nabla q|^{2} \mathrm{~d} x \mathrm{~d} t+s^{3} \lambda^{4} \int_{Q} \varphi^{3} \mathrm{e}^{-2 s \eta}|q|^{2} \mathrm{~d} x \mathrm{~d} t \\
& \leq C(Q, \omega, c)\left[s^{3} \lambda^{4} \int_{\omega_{T}} \varphi^{3} \mathrm{e}^{-2 s \eta}|q|^{2} \mathrm{~d} x \mathrm{~d} t+\int_{Q} \mathrm{e}^{-2 s \eta}\left|L^{(*)} q\right|^{2} \mathrm{~d} x \mathrm{~d} t\right] .
\end{aligned}
$$

(Proof: extend theorem 3.3 of [17, page 629] to the case $q \in D\left(L^{(*)}\right)$; see details in appendix 6.1).

- If $f \in\left(L^{2}(Q)\right)^{n}$ and $\mathcal{L} q=\operatorname{div}_{x} f \in H^{-1}(Q)$ in the sense of appendix, then we have

$$
s^{3} \lambda^{4} \int_{Q} \varphi^{3} \mathrm{e}^{-2 s \eta}|q|^{2} \leq C(Q, \omega, c)\left[s^{2} \lambda^{2} \int_{Q} \varphi^{2} \mathrm{e}^{-2 s \eta}|f|^{2}+s^{3} \lambda^{4} \int_{\omega_{T}} \varphi^{3} \mathrm{e}^{-2 s \eta}|q|^{2}\right] .
$$

(Proof in appendix).

Case 2-

- If $q \in D\left(L^{(*)}\right)$ then we have

$$
\begin{aligned}
& s \lambda^{2} \int_{Q} \sum_{j} \varphi_{j} \mathrm{e}^{-2 s \eta_{j}} \mid\left.\nabla q\right|^{2} \mathrm{~d} x \mathrm{~d} t+s^{3} \lambda^{4} \int_{Q} \sum_{j} \varphi_{j}^{3} \mathrm{e}^{-2 s \eta_{j}}|q|^{2} \mathrm{~d} x \mathrm{~d} t \\
& \leq C(Q, \omega, c)\left[s^{3} \lambda^{4} \int_{\omega_{T}} \sum_{j} \varphi_{j}^{3} \mathrm{e}^{-2 s \eta_{j}}|q|^{2} \mathrm{~d} x \mathrm{~d} t+\int_{Q} \sum_{j} \mathrm{e}^{-2 s \eta_{j}}\left|L^{(*)} q\right|^{2} \mathrm{~d} x \mathrm{~d} t\right] .
\end{aligned}
$$

(Proof: extend theorem 3.4 of [17, page 639] to the case $q \in D\left(L^{(*)}\right)$; see details in appendix 6.1).

- If $f \in\left(L^{2}(Q)\right)^{n}$ and $\mathcal{L} q=\operatorname{div}_{x} f \in H^{-1}(Q)$ in the sense of appendix, then we have

$$
s^{3} \lambda^{4} \int_{Q} \sum_{j} \varphi_{j}^{3} \mathrm{e}^{-2 s \eta_{j}}|q|^{2} \leq C(Q, \omega, c)\left[s^{2} \lambda^{2} \int_{Q} \sum_{j} \varphi_{j}^{2} \mathrm{e}^{-2 s \eta_{j}}|f|^{2}+s^{3} \lambda^{4} \int_{\omega_{T}} \sum_{j} \varphi_{j}^{3} \mathrm{e}^{-2 s \eta_{j}}|q|^{2}\right]
$$

(Proof in appendix).

\section{Case 1-}

- If $q \in D\left(L^{(*)}\right)$ then we have

$$
\begin{aligned}
& s \lambda^{2} \int_{Q} \sum_{j} \varphi_{j} \mathrm{e}^{-2 s \eta_{j}} \mid\left.\nabla q\right|^{2} \mathrm{~d} x \mathrm{~d} t+s^{3} \lambda^{4} \int_{Q} \sum_{j} \varphi^{3} \mathrm{e}^{-2 s \eta_{j}}|q|^{2} \mathrm{~d} x \mathrm{~d} t \\
& \leq C(Q, \gamma, c)\left[s \lambda \int_{\gamma_{T}} \sum_{j} \varphi_{j} \mathrm{e}^{-2 s \eta_{j}}\left|\partial_{n} q\right|^{2} \mathrm{~d} \sigma \mathrm{d} t+\int_{Q} \sum_{j} \mathrm{e}^{-2 s \eta_{j}}\left|L^{(*)} q\right|^{2} \mathrm{~d} x \mathrm{~d} t\right] .
\end{aligned}
$$

(Proof: apply [15, Theorem 2.3] with $g=0$ and then extend it to the case $q \in D\left(L^{(*)}\right)$; see details in appendix 6.1. Notice that, since $g=0$, we don't need here the assumption $c_{1}-c_{0} \geq \Delta>0$ of [15, Theorem 2.3]). 
- If $f \in\left(L^{2}(Q)\right)^{n} \cap\left(L^{2}\left(t_{0}, T ; H^{1}\left(V_{\gamma}\right)\right)\right)^{n}$, where $V_{\gamma}$ denotes a neighbourhood of $\gamma$, if $q \in L^{2}(Q) \cap L^{2}\left(t_{0}, T ; H^{2}\left(V_{\gamma}\right)\right)$ and $\mathcal{L} q=\operatorname{div}_{x} f \in H^{-1}(Q)$ in the sense of appendix, then we have

$$
\begin{aligned}
& s^{3} \lambda^{4} \int_{Q} \sum_{j} \varphi_{j}^{3} \mathrm{e}^{-2 s \eta_{j}}|q|^{2} \mathrm{~d} x \mathrm{~d} t \leq C(Q, \gamma, c)[ \\
& \left.s^{2} \lambda^{2} \int_{Q} \sum_{j} \varphi_{j}^{2} \mathrm{e}^{-2 s \eta_{j}}|f|^{2} \mathrm{~d} x \mathrm{~d} t+s \lambda \int_{\gamma_{T}} \sum_{j} \varphi_{j} \mathrm{e}^{-2 s \eta_{j}}\left|f \cdot n+c_{0} \partial_{n} q\right|^{2} \mathrm{~d} \sigma \mathrm{d} t\right] .
\end{aligned}
$$

(Proof in appendix).

\section{Inverse problem for case 3}

This section is devoted to the resolution of the inverse problem for case 3 . We prove (1.5) which implies uniqueness for $c$ in case 3 with only one measurement.

\subsection{A stationary problem}

This is the second key ingredient for solving our inverse problem. We look for $L^{2}(\Omega)$ stability for $c(x)$ solution of the following first-order partial differential equation:

$$
-\operatorname{div}(c \nabla u)=f \text { in } \Omega,
$$

where $u \in H^{1}(\Omega), f \in L^{2}(\Omega)$. So we consider too $c^{\prime}(x)$ solution of $-\operatorname{div}\left(c^{\prime} \nabla u^{\prime}\right)=f^{\prime}$, where $u^{\prime} \in H^{1}(\Omega), f^{\prime} \in L^{2}(\Omega)$.

We denote constants which depend on $Q, T, T^{\prime}, c, c^{\prime}$ or on $\omega$ simply by " $C$ ". Constants depending on $s$ and $\lambda$ are denoted by $C(s, \lambda)$. We set the following notations where $q$ stands for a function:

$\eta$ (respectively $\varphi$ ) replaces $\eta\left(T^{\prime}\right)$ (respectively $\varphi\left(T^{\prime}\right)$ ); the geometrical objects associated with $c^{\prime}$ keep the notation given for $c$, but with an additional prime. That is, the interface $c^{\prime}$ is denoted $S^{\prime}$; the unit normal $n^{\prime}=n_{1}^{\prime}=-n_{0}^{\prime}$ on $S^{\prime}$ points towards $\Omega_{0}^{\prime}$, etc; the jump of $q$ on $S^{\prime}$ is $[q]^{\prime}:=\left(q_{\Omega_{1}}\right)_{\mid S^{\prime}}-\left(q_{\Omega_{0}}\right)_{\mid S^{\prime}} ; \Omega_{j k}:=\Omega_{j} \cap \Omega_{k}^{\prime} ; q_{j k} \equiv q_{\mid \Omega_{j k}}$; $\Omega^{\prime \prime}:=\Omega \backslash\left(S \cup S^{\prime}\right)=\cup_{j, k=0,1} \Omega_{j k} ; E_{ \pm}:=\left\{x \in S \cap S^{\prime} ; n(x)= \pm n^{\prime}(x)\right\}, E:=E_{-} \cup E_{+}$.

We set $\xi:=c-c^{\prime}$. The main result of this part is

Theorem 3.1 Under the assumptions:

(HS3a): $|\nabla \beta \cdot \nabla u| \geq \delta>0$ in $\Omega^{\prime \prime} \backslash \omega$,

(HS3b): $u_{\mid \Omega_{j}} \in \mathcal{C}^{2}\left(\overline{\Omega_{j}}\right), u_{\mid \Omega_{j}^{\prime}}^{\prime} \in \mathcal{C}^{2}\left(\overline{\Omega_{j}^{\prime}}\right), j=0,1$,

we have the following estimate:

$$
\begin{aligned}
\delta^{2} \int_{\Omega} \varphi^{3} \mathrm{e}^{-2 s \eta}|\xi|^{2} \mathrm{~d} x \leq & C s^{-2} \lambda^{-2} \int_{\Omega} \varphi \mathrm{e}^{-2 s \eta}\left|f-f^{\prime}\right|^{2}+C M_{1}^{2} \int_{\omega} \varphi^{3} \mathrm{e}^{-2 s \eta}|\xi|^{2} \mathrm{~d} x \\
& +C(s, \lambda)\left\|\nabla\left(u-u^{\prime}\right)\right\|_{L^{\infty}(\Omega)},
\end{aligned}
$$

if $\lambda \geq \lambda_{0}$ and $s \geq s_{0}\left(\lambda, \delta, M_{2}\right)$, where we set

$$
M_{j}:=\max \left(\|u\|_{W^{j, \infty}\left(\Omega^{\prime \prime}\right)},\left\|u^{\prime}\right\|_{W^{j, \infty}\left(\Omega^{\prime \prime}\right)}\right), j=1,2 .
$$


Proof - Since $\partial \Omega^{\prime \prime}$ is not necessarily lipschitzian, the Gauss-Green formula in $\Omega^{\prime \prime}$ is replaced by the De Giorgi-Federer formula and can be used as follows. Let $q$ be a vector field in $\mathbb{R}^{n}$ such that $q_{j k} \equiv q_{\mid \Omega_{j k}} \in \mathcal{C}^{1}\left(\overline{\Omega_{j k}}\right)$. Then we have:

$\int_{\Omega^{\prime \prime}} \operatorname{div} q=\int_{\partial \Omega} q \cdot n \mathrm{~d} \sigma+\int_{S^{\prime} \backslash S}[q]^{\prime} \cdot n^{\prime} \mathrm{d} \sigma+\int_{S \backslash S^{\prime}}[q] \cdot n \mathrm{~d} \sigma+\int_{E}[q] \cdot n \mathrm{~d} \sigma$.

Note that $\int_{E}[q] \cdot n=\int_{E}[q]^{\prime} \cdot n^{\prime}$, since $[q]^{\prime}= \pm[q]$ on $E_{ \pm}$.

We denote by $n^{\prime \prime}$ the unit normal on $\Gamma^{\prime \prime} \equiv \partial^{*} \Omega^{\prime \prime}=\Gamma \cup\left(S^{\prime} \backslash S\right) \cup\left(S \backslash S^{\prime}\right) \cup E$, which is determined by $n^{\prime \prime}=n$ on $S \cup \Gamma, n^{\prime \prime}=n^{\prime}$ on $S^{\prime} \backslash E$. The jump of $q$ on $\Gamma^{\prime \prime}$ is denoted $[q]^{\prime \prime}$ and is determined by $[q]^{\prime \prime}=[q]$ on $S$ (undetermined on $S \cap S^{\prime} \backslash E$ which is d $\sigma$-negligible), and $[q]^{\prime \prime}=[q]^{\prime}$ on $S^{\prime} \backslash E$ (undetermined on $S^{\prime} \cap S \backslash E$ which is d $\sigma$-negligible too). Then we obtain:

$$
\int_{\Omega^{\prime \prime}} \operatorname{div} q=\int_{\Gamma^{\prime \prime}}[q]^{\prime \prime} \cdot n^{\prime \prime} \mathrm{d} \sigma
$$

with $[q]^{\prime \prime}:=q$ on $\Gamma$.

We prove (3.3) as follows. We denote by $\Gamma_{j k} \equiv \partial^{*} \Omega_{j k}$ the reduced boundary of $\Omega_{j k}$ :

$$
\begin{aligned}
\int_{\Omega^{\prime \prime}} \operatorname{div} q & =\sum_{j k} \int_{\Omega_{j k}} \operatorname{div} q_{j k}=\sum_{j k} \int_{\Gamma_{j k}} q_{j k} \cdot n \\
& =\sum_{j k} \int_{\Gamma_{j k} \cap \Gamma} q_{j k} \cdot n+\sum_{j k} \int_{\Gamma_{j k} \cap S} q_{j k} \cdot n_{j}+\sum_{j k} \int_{\Gamma_{j k} \cap S^{\prime}} q_{j k} \cdot n_{k}^{\prime}+\sum_{j k} \int_{\Gamma_{j k} \cap E} q_{j k} \cdot n_{j}
\end{aligned}
$$

with $n_{j}=n_{k}^{\prime}$ on $\Gamma_{j k} \cap E$ (note that a point $x \in E$ such that $n_{j}(x)=-n_{k}^{\prime}(x)$ belongs to $\left.\partial \Omega_{j k} \backslash \Gamma_{j k}\right)$. The boundary $\Gamma$ (respectively $S^{\prime} \backslash S, S \backslash S^{\prime}, E_{ \pm}$) is partitioned by the $\Gamma_{j k} \cap \Gamma$ (respectively $\left.\Gamma_{j k} \cap\left(S^{\prime} \backslash S\right), \Gamma_{j k} \cap\left(S \backslash S^{\prime}\right), \Gamma_{j k} \cap E_{ \pm}\right)$. This implies the conclusion as well as the following formula:

$$
\int_{\Omega^{\prime \prime}} \phi \operatorname{div} q=\int_{\Gamma^{\prime \prime}}[\phi q]^{\prime \prime} \cdot n^{\prime \prime} \mathrm{d} \sigma-\int_{\Omega^{\prime \prime}} \nabla \phi \cdot q,
$$

when $\phi_{j k}, q_{j k} \in \mathcal{C}^{1}\left(\overline{\Omega_{j k}}\right)$.

If $q$ is a function in $\Omega$, we set $q_{s} \equiv \varphi^{\frac{1}{2}} \mathrm{e}^{-s \eta} q$.

We have:

$$
\begin{aligned}
& P u \equiv-\operatorname{div}\left(c_{s} \nabla u\right)-s \nabla \eta\left(T^{\prime}\right) c_{s} \cdot \nabla u=f_{s}-\frac{1}{2} c_{s} \frac{\nabla \varphi\left(T^{\prime}\right)}{\varphi\left(T^{\prime}\right)} \cdot \nabla u \\
& P^{\prime} u^{\prime} \equiv-\operatorname{div}\left(c_{s}^{\prime} \nabla u^{\prime}\right)-s \nabla \eta\left(T^{\prime}\right) c_{s}^{\prime} \cdot \nabla u^{\prime}=f_{s}^{\prime}-\frac{1}{2} c_{s}^{\prime} \frac{\nabla \varphi\left(T^{\prime}\right)}{\varphi\left(T^{\prime}\right)} \cdot \nabla u^{\prime} .
\end{aligned}
$$

Note that $P u, P^{\prime} u^{\prime} \in L^{2}(\Omega)$. We set $M_{s}:=P u-P^{\prime} u^{\prime}$ and $I\left(u, u^{\prime}\right):=\left\|M_{s}\right\|_{L^{2}(\Omega ; c(x) \mathrm{d} x)}^{2}$. Thus we have

$$
\begin{aligned}
M_{s} & =f_{s}-f_{s}^{\prime}-\frac{1}{2} \lambda \nabla \beta \cdot\left(\xi_{s} \cdot \nabla u+c_{s}^{\prime} \nabla\left(u-u^{\prime}\right)\right) \\
& =-\operatorname{div}\left(c_{s} \nabla u-c_{s}^{\prime} \nabla u^{\prime}\right)-s \nabla \eta\left(T^{\prime}\right) \cdot\left(c_{s} \nabla u-c_{s}^{\prime} \nabla u^{\prime}\right) .
\end{aligned}
$$

On one hand, thanks to (3.6), we have:

$$
\begin{aligned}
I\left(u, u^{\prime}\right) & \leq 2 c_{\max }\left(\left\|f_{s}-f_{s}^{\prime}\right\|_{L^{2}(\Omega ; \mathrm{d} x)}^{2}+\lambda^{2} \int_{\Omega}\left|\xi_{s}\right|^{2}|\nabla \beta \cdot \nabla u|^{2}\right)+C \lambda^{2} \int_{\Omega} \varphi\left|\nabla\left(u-u^{\prime}\right)\right|^{2} \\
& \leq C\left(\left\|f_{s}-f_{s}^{\prime}\right\|^{2}+\lambda^{2}\|\nabla u\|_{L^{\infty}}^{2} \int_{\Omega}\left|\xi_{s}\right|^{2}+\lambda^{2} \int_{\Omega} \varphi\left|\nabla\left(u-u^{\prime}\right)\right|^{2}\right) .
\end{aligned}
$$


Uniqueness of discontinuous diffusion coefficients for the heat equation

On the other hand, thanks to $(3.7)$ and $(a+b)^{2} \geq a^{2}+2 a b$, we have:

$$
I\left(u, u^{\prime}\right) \geq s^{2} B_{1}+2 s B_{2},
$$

where

$$
\begin{aligned}
& B_{1}:=\int_{\Omega^{\prime \prime}}\left|\nabla \eta \cdot\left(c_{s} \nabla u-c_{s}^{\prime} \nabla u^{\prime}\right)\right|^{2} c(x) \mathrm{d} x, \\
& B_{2}:=\int_{\Omega^{\prime \prime}}\left(\operatorname{div}\left(c_{s}^{\prime} \nabla u^{\prime}\right)-\operatorname{div}\left(c_{s} \nabla u\right)\right) \nabla \eta \cdot\left(c_{s}^{\prime} \nabla u^{\prime}-c_{s} \nabla u\right) c(x) \mathrm{d} x .
\end{aligned}
$$

Thanks to $(a+b)^{2} \geq \frac{1}{2} a^{2}-b^{2}$ and (HS3a), we have:

$$
\begin{aligned}
B_{1} & =\lambda^{2} \int_{\Omega^{\prime \prime}} \varphi^{2}\left|\nabla \beta \cdot\left(\xi_{s} \nabla u+c_{s}^{\prime} \nabla\left(u-u^{\prime}\right)\right)\right|^{2} c(x) \mathrm{d} x \\
& \geq c_{\min } \lambda^{2} \int_{\Omega^{\prime \prime} \backslash \omega} \varphi^{2}\left(\frac{1}{2} \xi_{s}^{2}|\nabla \beta \cdot \nabla u|^{2}-\left(c_{s}^{\prime}\right)^{2}\left|\nabla \beta \cdot \nabla\left(u-u^{\prime}\right)\right|^{2}\right) \mathrm{d} x \\
& \geq \frac{1}{2} c_{\text {min }} \delta^{2} \lambda^{2} \int_{\Omega \backslash \omega} \varphi^{2}\left|\xi_{s}\right|^{2} \mathrm{~d} x-C \lambda^{2} \int_{\Omega} \varphi^{3} \mathrm{e}^{-2 s \eta}\left|\nabla\left(u-u^{\prime}\right)\right|^{2} \mathrm{~d} x .
\end{aligned}
$$

Let us give a lower bound for $B_{2}$ by using (3.5).

Let $\phi:=-\xi_{s} c \nabla \eta \cdot \nabla u, q:=\left(c_{s}^{\prime} \nabla u^{\prime}-c_{s} \nabla u\right)$, we have $B_{2}=B_{20}+X_{1}$ with

$$
B_{20}:=\int_{\Omega^{\prime \prime}} \phi \operatorname{div} q \text {, and } X_{1}:=-\int_{\Omega^{\prime \prime}} c c_{s}^{\prime} \nabla \eta \cdot \nabla\left(u-u^{\prime}\right) \operatorname{div} q \text {. }
$$

Since $|\operatorname{div} q| \leq C s \lambda \varphi^{3 / 2}\left(M_{2}\right)$ we obtain

$$
\begin{aligned}
\left|X_{1}\right| & \leq C M_{2} \int_{\Omega^{\prime \prime}} s \lambda^{2} \varphi^{3} \mathrm{e}^{-2 s \eta}\left|\nabla\left(u-u^{\prime}\right)\right| \\
& \leq C(s, \lambda) M_{2}\left\|\nabla\left(u-u^{\prime}\right)\right\|_{L^{\infty}(\Omega)} .
\end{aligned}
$$

Now, we consider $B_{20}$. Note that the functions $\phi$ and $q$ satisfy $\phi_{j k}, q_{j k} \in \mathcal{C}^{1}\left(\overline{\Omega_{j k}}\right)$. Let $p:=\phi q$. We determine the jump of $[p]^{\prime \prime}$ on $\Gamma^{\prime \prime}$. Note that $\left[q^{\prime \prime}\right] \cdot n^{\prime \prime}=0$ thanks to the properties of the weight functions. Thus we have $\left[p^{\prime \prime}\right] \cdot n^{\prime \prime}=[\phi]^{\prime \prime}\left(q \cdot n^{\prime \prime}\right)$.

* On $S \backslash S^{\prime}$. Since $\eta$ is constant on $S,(\nabla \eta)_{\mid S}=\partial_{n} \eta n$, then

$$
[p] \cdot n=\left(c_{s} \partial_{n} \eta\right)\left(c \partial_{n} u-c^{\prime} \partial_{n} u^{\prime}\right)\left[\xi_{s} \partial_{n} u\right] .
$$

Note that $\left[\xi_{s} \partial_{n} u\right]=\left[-c_{s}^{\prime} \partial_{n} u\right]=-\left[c_{s}^{\prime}\left(\partial_{n} u-\partial_{n} u^{\prime}\right)\right]$. Hence

$$
[p] \cdot n=-\left(c_{s} \partial_{n} \eta\right)\left(c \partial_{n} u-c^{\prime} \partial_{n} u^{\prime}\right) c_{s}^{\prime}\left[\partial_{n}\left(u-u^{\prime}\right)\right] .
$$

* On $S^{\prime} \backslash S$. Since $\left[\xi_{s}\right]^{\prime}=-\left[c_{s}^{\prime}\right]^{\prime}$, then $[p]^{\prime} \cdot n^{\prime}=-(c \nabla \eta \cdot \nabla u)\left(c_{s} \partial_{n^{\prime}} u-c_{s}^{\prime} \partial_{n^{\prime}} u^{\prime}\right)\left[c_{s}^{\prime}\right]^{\prime}$.

Note that $c_{s} \partial_{n^{\prime}} u\left[c_{s}^{\prime}\right]^{\prime}=c_{s}\left[c_{s}^{\prime} \partial_{n^{\prime}}\left(u-u^{\prime}\right)\right]^{\prime}$. On the other hand we have $c^{\prime 2}=\left(c_{0}^{\prime}+c_{1}^{\prime}\right) c^{\prime}-c_{0}^{\prime} c_{1}^{\prime}$ and so

$$
\left[c^{\prime 2} \partial_{n^{\prime}} u^{\prime}\right]^{\prime}=\left[-c_{0}^{\prime} c_{1}^{\prime} \partial_{n^{\prime}} u^{\prime}\right]^{\prime}=c_{0}^{\prime} c_{1}^{\prime}\left[\partial_{n^{\prime}}\left(u-u^{\prime}\right)\right]^{\prime} .
$$

Hence we obtain

$$
[p]^{\prime} \cdot n^{\prime}=(c \nabla \eta \cdot \nabla u)\left[\left(c_{0, s}^{\prime} c_{1}^{\prime}-c_{s} c_{s}^{\prime}\right)\left(\partial_{n^{\prime}}\left(u-u^{\prime}\right)\right]^{\prime} .\right.
$$


Uniqueness of discontinuous diffusion coefficients for the heat equation

* On $E$. This is similar to the case $S \backslash S^{\prime}$. Hence

$$
[p] \cdot n=-\left(c_{s} \partial_{n} \eta\right)\left(c \partial_{n} u-c^{\prime} \partial_{n} u^{\prime}\right)\left[c_{s}^{\prime} \partial_{n}\left(u-u^{\prime}\right)\right] .
$$

If we set $K(s, \lambda):=\lambda \int_{\Gamma^{\prime \prime}} \varphi^{2} \mathrm{e}^{-2 s \eta} \mathrm{d} \sigma$, then the three previous cases show the following estimate:

$$
\left\|[p]^{\prime \prime} \cdot n^{\prime \prime}\right\|_{L^{\infty}\left(\Gamma^{\prime \prime} \backslash \Gamma\right)} \leq C K(s, \lambda) M_{1}\left\|\nabla\left(u-u^{\prime}\right)\right\|_{L^{\infty}(\Omega)}
$$

* On $\Gamma$.

$$
p \cdot n=\xi_{s}^{2}\left(c \partial_{n} \eta\right)\left(\partial_{n} u\right)^{2}+\xi_{s} c_{s}^{\prime}\left(c \partial_{n} \eta\right)\left(\partial_{n} u\right) \partial_{n}\left(u-u^{\prime}\right) .
$$

Furthermore the last term of the right hand side in (3.12) can be estimated as in (3.11).

We resume the calculations for $B_{2}$ :

$$
\begin{aligned}
B_{2} & =X_{1}+B_{21}+B_{4}, \\
B_{4} & :=-\int_{\Omega^{\prime \prime}} \nabla \phi q . \\
B_{21} & :=\int_{\Gamma^{\prime \prime}}[p]^{\prime \prime} \cdot n^{\prime \prime}=X_{2}+B_{3}+X_{3}, \\
X_{2} & :=\int_{\Gamma^{\prime \prime} \backslash \Gamma}[p]^{\prime \prime} \cdot n^{\prime} \geq-C(s, \lambda) M_{1}\left\|\nabla\left(u-u^{\prime}\right)\right\|_{L^{\infty}(\Omega)}, \\
B_{3} & :=\int_{\Gamma} \xi_{s}^{2}\left(c \partial_{n} \eta\right)\left(\partial_{n} u\right)^{2} \mathrm{~d} x=-\lambda \int_{\Gamma} \varphi \partial_{n} \beta\left(\partial_{n} u\right)^{2}\left|\xi_{s}\right|^{2} c(x) \mathrm{d} x \\
& \geq 0 ; \\
X_{3} & :=\int_{\Gamma} \xi_{s} c_{s}^{\prime}\left(c \partial_{n} \eta\right)\left(\partial_{n} u\right) \partial_{n}\left(u-u^{\prime}\right) \mathrm{d} x \\
& \geq-C(s, \lambda) M_{1}\left\|\nabla\left(u-u^{\prime}\right)\right\|_{L^{\infty}(\Omega)} .
\end{aligned}
$$

Let us compute $B_{4}$. In $\Omega^{\prime \prime}$ we have

$$
\begin{aligned}
-\phi= & \sum_{l} c \partial_{l} \eta \cdot \xi_{s} \partial_{l} u, \\
-\partial_{i} \phi= & \sum_{l}\left\{\partial_{i}\left(c \partial_{l} \eta \partial_{l} u\right) \xi_{s}+c \partial_{l} \eta \partial_{l} u \partial_{i} \xi_{s}\right\}, \\
-\nabla \phi \cdot q= & -c_{s}^{\prime} \nabla\left(\xi_{s} c \nabla \eta \cdot \nabla u\right) \cdot \nabla\left(u-u^{\prime}\right)-\xi_{s}^{2} \nabla(c \nabla \eta \cdot \nabla u) \cdot \nabla u \\
& -\frac{1}{2}(c \nabla \eta \cdot \nabla u)\left(\nabla \xi_{s}^{2} \cdot \nabla u\right) .
\end{aligned}
$$

Thus $B_{4}=X_{4}+B_{5}+\frac{1}{2} B_{6}$ with

$$
\begin{aligned}
& X_{4}:=-\int_{\Omega^{\prime \prime}} c_{s}^{\prime} \nabla\left(\xi_{s} c \nabla \eta \cdot \nabla u\right) \cdot \nabla\left(u-u^{\prime}\right), \\
& B_{5}:=-\int_{\Omega^{\prime \prime}} \nabla u \cdot \nabla(c \nabla \eta \cdot \nabla u)\left|\xi_{s}\right|^{2}, \\
& B_{6}:=-\int_{\Omega^{\prime \prime}}(c \nabla \eta \cdot \nabla u)\left(\nabla u \cdot \nabla\left(\xi_{s}^{2}\right)\right) \mathrm{d} x .
\end{aligned}
$$

Since $\nabla(c \nabla \eta \cdot \nabla u)=-\lambda \varphi \nabla(c \nabla \beta \cdot \nabla u)-\lambda^{2} \varphi(c \nabla \beta \cdot \nabla u) \nabla \beta$, then we have $B_{5}=B_{51}+B_{52}$ with

$B_{51}:=\lambda \int_{\Omega^{\prime \prime}} \varphi \nabla(c \nabla \beta \cdot \nabla u) \cdot \nabla u\left|\xi_{s}\right|^{2}$ and $B_{52}:=\lambda^{2} \int_{\Omega^{\prime \prime}} \varphi(\nabla \beta \cdot \nabla u)^{2}\left|\xi_{s}\right|^{2} c(x) \mathrm{d} x$. 
Uniqueness of discontinuous diffusion coefficients for the heat equation

We treat the integral $B_{6}$ as we did for $B_{2}$. From now we set:

$$
q:=(c \nabla \eta \cdot \nabla u) \nabla u, \phi:=\xi_{s}^{2} \text { and } p:=\phi q .
$$

Then we have $B_{6}=B_{7}+B_{8}$ with

$$
B_{7}:=-\int_{\Gamma^{\prime \prime}}[p]^{\prime \prime} \cdot n^{\prime \prime} \text { and } B_{8}:=\int_{\Omega^{\prime \prime}} \phi \operatorname{div} q \mathrm{~d} x .
$$

We determine the jump $[p]^{\prime \prime}$ on $\Gamma^{\prime \prime}$.

* On $S \backslash S^{\prime}$. We have $[p] \cdot n=\left(c \partial_{n} \eta\right)\left[\xi_{s}^{2}\left(\partial_{n} u\right)^{2}\right]$. Since

$$
\begin{aligned}
{\left[\xi_{s}^{2}\left(\partial_{n} u\right)^{2}\right] } & =\left[\left(-2 c_{s} c_{s}^{\prime}+\left(c_{s}^{\prime}\right)^{2}\right)\left(\partial_{n} u\right)^{2}\right]=-2\left(c_{s} \partial_{n} u\right) c_{s}^{\prime}\left[\partial_{n} u\right]+\left(c_{s}^{\prime}\right)^{2}\left[\left(\partial_{n} u\right)^{2}\right] \\
& =-2\left(c_{s} \partial_{n} u\right) c_{s}^{\prime}\left[\partial_{n}\left(u-u^{\prime}\right)\right]+\left(c_{s}^{\prime}\right)^{2}\left[\left(\partial_{n} u\right)^{2}-\left(\partial_{n} u^{\prime}\right)^{2}\right] \\
& =-c_{s}^{\prime}\left[\left(2 \xi_{s} \partial_{n} u+c_{s}^{\prime} \partial_{n}\left(u-u^{\prime}\right)\right)\left(\partial_{n}\left(u-u^{\prime}\right)\right)\right]
\end{aligned}
$$

then we obtain

$$
[p] \cdot n=-c_{s}^{\prime}\left(c \partial_{n} \eta\right)\left[\left(2 \xi_{s} \partial_{n} u+c_{s}^{\prime} \partial_{n}\left(u-u^{\prime}\right)\right)\left(\partial_{n}\left(u-u^{\prime}\right)\right)\right] .
$$

* On $S^{\prime} \backslash S$. We have $[p]^{\prime} \cdot n^{\prime}=(c \nabla \eta \nabla u) \partial_{n^{\prime}} u\left[\xi_{s}^{2}\right]^{\prime}$. Since $\left[\xi_{s}^{2}\right]^{\prime}=-2 c_{s}\left[c_{s}^{\prime}\right]^{\prime}+\left[\left(c_{s}^{\prime}\right)^{2}\right]^{\prime}$ then

$$
\partial_{n^{\prime}} u\left[\xi_{s}^{2}\right]^{\prime}=\left[c_{s}^{\prime}\left(-2 c_{s}+c_{s}^{\prime}\right) \partial_{n^{\prime}}\left(u-u^{\prime}\right)\right]^{\prime}+\left[\left(c_{s}^{\prime}\right)^{2} \partial_{n^{\prime}} u^{\prime}\right]^{\prime} .
$$

Thanks to $c^{\prime 2}=\left(c_{0}^{\prime}+c_{1}^{\prime}\right) c^{\prime}-c_{0}^{\prime} c_{1}^{\prime}$, we have

$$
\left[\left(c^{\prime}\right)^{2} \partial_{n^{\prime}} u^{\prime}\right]^{\prime}=-c_{0}^{\prime} c_{1}^{\prime}\left[\partial_{n^{\prime}} u^{\prime}\right]^{\prime}=c_{0}^{\prime} c_{1}^{\prime}\left[\partial_{n^{\prime}}\left(u-u^{\prime}\right)\right]^{\prime}
$$

Hence

$$
[p] \cdot n=(c \nabla \eta \nabla u)\left[\left(c_{s}^{\prime}\left(-2 c_{s}+c_{s}^{\prime}\right)+c_{0, s}^{\prime} c_{1, s}^{\prime}\right) \partial_{n^{\prime}}\left(u-u^{\prime}\right)\right]^{\prime} .
$$

* On $E$. This is similar to the case $S \backslash S^{\prime}$, with the difference that $\left[c^{\prime}\right] \neq 0$. Hence $[p]^{\prime} \cdot n^{\prime}=-\left(c \partial_{n} \eta\right)\left[c_{s}^{\prime}\left(2 \xi_{s} \partial_{n} u+c_{s}^{\prime} \partial_{n}\left(u-u^{\prime}\right)\right)\left(\partial_{n}\left(u-u^{\prime}\right)\right)\right]$.

The three previous cases show that (3.11) holds (with $p$ defined as above).

* On $\Gamma$.

$$
p \cdot n=-\lambda \varphi \xi_{s}^{2}\left(c \partial_{n} \beta\right)\left(\partial_{n} u\right)^{2} \geq 0 .
$$

Thus we have $B_{7}=B_{71}+X_{5}$ with

$$
B_{71}:=-\int_{\Gamma}[p]^{\prime \prime} \cdot n^{\prime \prime}=-B_{3} \text { and } X_{5}:=-\int_{\Gamma^{\prime \prime} \backslash \Gamma}[p]^{\prime \prime} \cdot n^{\prime \prime}
$$

And so:

$$
\left|X_{5}\right| \leq C(s, \lambda) M_{1}\left\|\nabla\left(u-u^{\prime}\right)\right\|_{L^{\infty}(\Omega)} .
$$

Note that the parts of $X_{2}$ and $X_{5}$ which bring about the non lipschitzian estimate (1.2) are integrals on $\left(S \backslash S^{\prime}\right) \cup\left(S^{\prime} \backslash S\right)$.

If we consider $X_{4}, B_{5}, B_{8}$, we obtain

$$
\begin{aligned}
\left|X_{4}\right| & \leq C(s, \lambda) M_{2}\left\|\nabla\left(u-u^{\prime}\right)\right\|_{L^{\infty}(\Omega)}, \\
\left|B_{5}\right|+\left|B_{8}\right| & \leq C\left(\lambda^{2} M_{1}^{2}+\lambda M_{2}^{2}\right) \int_{\Omega^{\prime \prime}} \varphi\left|\xi_{s}\right|^{2} .
\end{aligned}
$$


Uniqueness of discontinuous diffusion coefficients for the heat equation

Since $B_{3}+\frac{1}{2} B_{71}=\frac{1}{2} B_{3} \geq 0$ then we have

$$
\begin{aligned}
B_{2} & \geq-\left|B_{5}\right|-\left|B_{8}\right|-\sum_{j=1}^{5}\left|X_{j}\right| \\
& \geq-C\left(\lambda^{2} M_{1}^{2}+\lambda M_{2}^{2}\right) \int_{\Omega^{\prime \prime}} \varphi\left|\xi_{s}\right|^{2}-C(s, \lambda) M_{2}\left\|\nabla\left(u-u^{\prime}\right)\right\|_{L^{\infty}(\Omega)}
\end{aligned}
$$

From (3.8), (3.9) (3.10), (3.15) we obtain

$$
\begin{aligned}
C\left(\left\|f_{s}-f_{s}^{\prime}\right\|^{2}\right. & \left.+\lambda^{2} M_{1}^{2} \int_{\Omega}\left|\xi_{s}\right|^{2}\right)+C(s, \lambda)\left\|\nabla\left(u-u^{\prime}\right)\right\|_{L^{\infty}(\Omega)}^{2} \\
\geq I\left(u, u^{\prime}\right) \geq & C \delta^{2} s^{2} \lambda^{2} \int_{\Omega \backslash \omega} \varphi^{2}\left|\xi_{s}\right|^{2} \mathrm{~d} x-C M_{1}^{2} s^{2} \lambda^{2} \int_{\omega} \varphi^{2}\left|\xi_{s}\right|^{2} \mathrm{~d} x \\
& \quad C\left(\lambda^{2} M_{1}^{2}+\lambda M_{2}^{2}\right) s \int_{\Omega} \varphi\left|\xi_{s}\right|^{2}-C(s, \lambda)\left\|\nabla\left(u-u^{\prime}\right)\right\|_{L^{\infty}(\Omega)} .
\end{aligned}
$$

Hence (3.1) holds if $s \geq s_{0}\left(\lambda, \delta, M_{2}\right)$ sufficiently large and $\delta \leq 1$ (non restrictive).

Remark 3.1 We can set $s_{0}\left(\lambda, \delta, M_{2}\right)=C(\lambda) M_{2}^{2} \delta^{-2}$ for some constant $C(\lambda)>0$, where $M_{2}$ is defined by (3.2).

\subsection{The non stationary problem for case 3}

For simplicity we fix $T^{\prime}=\frac{t_{0}+T}{2}$, but this is not restrictive, since it means insignificant modifications for the weight functions.

We consider in $\Omega$ two diffusion coefficients $c(x)$ and $c^{\prime}(x)$ associated respectively with the interfaces $S$ and $S^{\prime}$. Thus we have the following equalities

$$
\partial_{t} y-\operatorname{div}(c \nabla y)=\partial_{t} y^{\prime}-\operatorname{div}\left(c^{\prime} \nabla y^{\prime}\right)=H \chi_{\omega} \text { in } Q,
$$

with the boundary conditions:

$$
y(t, x)=y^{\prime}(t, x)=0 \text { on } \Sigma .
$$

Note that $y(0, \cdot)$ and $y^{\prime}(0, \cdot)$ (or $y\left(t_{0}, \cdot\right)$ and $\left.y^{\prime}\left(t_{0}, \cdot\right)\right)$ may be different. We prove that if functions $y, y^{\prime}$ coincide in $\Omega$ at time $T^{\prime} \in\left(t_{0}, T\right)$, then we have (under some additional assumptions) $c=c^{\prime}$ in $\Omega$.

We set $u(x):=y\left(T^{\prime}, x\right), u^{\prime}(x):=y^{\prime}\left(T^{\prime}, x\right)$ for $x \in \Omega$, and $v:=\partial_{t} y-\partial_{t} y^{\prime}, \xi:=c-c^{\prime}$.

Thus we have:

$$
\left\{\begin{aligned}
\partial_{t} v-\operatorname{div}(c \nabla v) & =\operatorname{div}\left(\xi \nabla \partial_{t} y^{\prime}\right) \text { in } Q, \\
v(t, x) & =0 \text { on } \Sigma
\end{aligned}\right.
$$

in the sense of appendix. Indeed for any $z \in D\left(L^{*}\right)$ we have

$$
\begin{aligned}
\int_{Q} \partial_{t} y L^{*} z & =\int_{Q} L \partial_{t} y z \\
\int_{Q} \partial_{t} y^{\prime} L^{*} z & =\int_{Q} \nabla \partial_{t} y^{\prime} \cdot c \nabla z-\int_{Q} \partial_{t} y^{\prime} \partial_{t} z \\
& =\int_{Q} \xi \nabla \partial_{t} y^{\prime} \cdot \nabla z+\int_{Q} c^{\prime} \nabla \partial_{t} y^{\prime} \cdot \nabla z+\int_{Q} \partial_{t}^{2} y^{\prime} z \\
& =\int_{Q} \xi \nabla \partial_{t} y^{\prime} \cdot \nabla z+\int_{Q} L^{\prime} \partial_{t} y^{\prime} z .
\end{aligned}
$$


Uniqueness of discontinuous diffusion coefficients for the heat equation

Notice that $\partial_{t} y \in D(L)$ and $\partial_{t} y^{\prime} \in D\left(L^{\prime}\right)$ since we choose $H$ such that $\partial_{t} H \in L^{2}(Q)$. Thus $v$ satisfies:

$\int_{Q} v L^{*} z=-\int_{Q} \xi \nabla \partial_{t} y^{\prime} \cdot \nabla z+\int_{Q}\left(L \partial_{t} y-L^{\prime} \partial_{t} y^{\prime}\right) z=-\int_{Q} \xi \nabla \partial_{t} y^{\prime} \cdot \nabla z$.

Moreover at time $T^{\prime} \in\left(t_{0}, T\right)$ we have in $\Omega$ :

$$
v\left(T^{\prime}, x\right)=\operatorname{div}\left(c \nabla u-c^{\prime} \nabla u^{\prime}\right)=\operatorname{div}\left(c^{\prime} \nabla\left(u-u^{\prime}\right)\right)+\operatorname{div}(\xi \nabla u) .
$$

We set

$$
A_{1}:=\int_{\Omega} \frac{1}{2}\left|v\left(T^{\prime}\right)\right|^{2} \varphi\left(T^{\prime}\right) \mathrm{e}^{-2 s \eta\left(T^{\prime}\right)} .
$$

An integration by parts gives

$$
A_{1}=\int_{t_{0}}^{T^{\prime}} \int_{\Omega} \frac{1}{2} \frac{\partial}{\partial t}\left(v^{2} \varphi \mathrm{e}^{-2 s \eta}\right)=A_{2}+A_{3},
$$

where we set

$$
A_{2}:=\int_{t_{0}}^{T^{\prime}} \int_{\Omega} v \partial_{t} v \varphi \mathrm{e}^{-2 s \eta} \text { and } A_{3}:=\int_{t_{0}}^{T^{\prime}} \int_{\Omega}\left(\frac{1}{2} \partial_{t} \varphi-s \varphi \partial_{t} \eta\right) \mathrm{e}^{-2 s \eta} v^{2} .
$$

Upper bounds of $A_{1^{-}}$We make the following assumption:

(H3c): $\nabla \partial_{t}^{k} y^{\prime} \in L^{2}\left(t_{0}, T ;\left(L^{\infty}(\Omega)\right)^{n}\right), k=1,2$.

We denote

$$
\begin{aligned}
M_{T, k} & :=\max _{k=1,2}\left\|\nabla \partial_{t}^{k} y^{\prime}\right\|_{L^{2}\left(t_{0}, T ;\left(L^{\infty}(\Omega)\right)^{n}\right)}, \\
M_{T} & :=\max _{k=1,2} M_{T, k} .
\end{aligned}
$$

From (2.1) we have $\left|A_{3}\right| \leq C\left(T, t_{0}\right) A_{3}^{\prime}$ with

$$
A_{3}^{\prime}:=\int_{Q} s \varphi^{3} \mathrm{e}^{-2 s \eta} v^{2}
$$

The Carleman estimate (2.5) with data in $H^{-1}$ implies:

$$
s^{2} \lambda^{4} A_{3}^{\prime} \leq C\left[A_{4}+s^{3} \lambda^{4} \int_{\omega_{T}} \varphi^{3} \mathrm{e}^{-2 s \eta}|v|^{2} \mathrm{~d} x \mathrm{~d} t\right]
$$

where we set $A_{4}:=\int_{Q^{\prime}} s^{2} \lambda^{2} \varphi^{2} \mathrm{e}^{-2 s \eta}\left|\nabla \partial_{t} y^{\prime}\right|^{2}|\xi|^{2} \mathrm{~d} x \mathrm{~d} t$. For $k \in \mathbb{N}$ we write:

$$
\varphi^{k} \mathrm{e}^{-2 s \eta}=\varphi^{k}\left(T^{\prime}\right) \mathrm{e}^{-2 s \eta\left(T^{\prime}\right)} \zeta^{k}(t) \mathrm{e}^{-2 s(\zeta(t)-1)},
$$

with $\zeta(t):=\left(t_{0}-T^{\prime}\right)\left(T-T^{\prime}\right)\left(t_{0}-t\right)^{-1}(T-t)^{-1} \geq 1$. Note that there exists $C_{k} \in \mathbb{R}$ independent of $s \geq 1$ and such that $\sup _{r \geq 1}\left(r^{k} \mathrm{e}^{-2 s(r-1)}\right) \leq C_{k}$. Thanks to (H3c) we obtain

$$
\begin{aligned}
A_{4} & \leq C_{2} \int_{\Omega} s^{2} \lambda^{2} \varphi^{2}\left(T^{\prime}\right) \mathrm{e}^{-2 s \eta\left(T^{\prime}\right)}|\xi|^{2}\left(\int_{t_{0}}^{T}\left|\nabla \partial_{t} y^{\prime}\right|^{2} \mathrm{~d} t\right) \mathrm{d} x \\
& \leq C_{2} M_{T, 1}^{2} \int_{\Omega} s^{2} \lambda^{2} \varphi^{2}\left(T^{\prime}\right) \mathrm{e}^{-2 s \eta\left(T^{\prime}\right)}|\xi|^{2} \mathrm{~d} x .
\end{aligned}
$$

Thus we obtain

$$
\left|A_{3}\right| \leq C\left(T, t_{0}\right) A_{3}^{\prime} \leq C\left[M_{T, 1}^{2} \int_{\Omega} \lambda^{-2} \varphi^{2}\left(T^{\prime}\right) \mathrm{e}^{-2 s \eta\left(T^{\prime}\right)}|\xi|^{2} \mathrm{~d} x+s \int_{\omega_{T}} \varphi^{3}|v|^{2} \mathrm{e}^{-2 s \eta} \mathrm{d} x \mathrm{~d} t\right] .
$$


Thanks to Minkovski's estimate we have $\left|A_{2}\right| \leq A_{3}^{\prime}+A_{21}$ with

$$
A_{21}:=\int_{Q^{\prime}} s^{-1} \varphi^{-1} \mathrm{e}^{-2 s \eta}\left|\partial_{t} v\right|^{2} .
$$

The function $w:=\partial_{t} v=\partial_{t}^{2} y-\partial_{t}^{2} y^{\prime}$ is solution of

$$
\begin{cases}\partial_{t} w-\operatorname{div}(c \nabla w) & =\operatorname{div}\left(\xi \nabla \partial_{t}^{2} y^{\prime}\right) \text { in } Q, \\ w(t, x) & =0 \text { on } \Sigma .\end{cases}
$$

Thanks to (H3c), an upper bound for $A_{21}$ is obtained as for $A_{3}^{\prime}$ by using the Carleman estimate (2.5) as follows:

$A_{21} \leq C\left[M_{T, 2}^{2} \int_{\Omega} s^{-2} \lambda^{-2} \varphi^{2}\left(T^{\prime}\right) \mathrm{e}^{-2 s \eta\left(T^{\prime}\right)}|\xi|^{2} \mathrm{~d} x+s^{-1} \int_{\omega_{T}} \varphi^{3}\left|\partial_{t} v\right|^{2} \mathrm{e}^{-2 s \eta} \mathrm{d} x \mathrm{~d} t\right]$.

So we obtain the following upper bound for $A_{1}$ :

$$
\begin{aligned}
A_{1} \leq & C\left[M_{T, 1}^{2} \int_{\Omega} \lambda^{-2} \varphi^{2}\left(T^{\prime}\right) \mathrm{e}^{-2 s \eta\left(T^{\prime}\right)}|\xi|^{2} \mathrm{~d} x+s \int_{\omega_{T}} \varphi^{3}|v|^{2} \mathrm{e}^{-2 s \eta} \mathrm{d} x \mathrm{~d} t\right. \\
& \left.+M_{T, 2}^{2} \int_{\Omega} s^{-2} \lambda^{-2} \varphi^{2}\left(T^{\prime}\right) \mathrm{e}^{-2 s \eta\left(T^{\prime}\right)}|\xi|^{2} \mathrm{~d} x+s^{-1} \int_{\omega_{T}} \varphi^{3}\left|\partial_{t} v\right|^{2} \mathrm{e}^{-2 s \eta} \mathrm{d} x \mathrm{~d} t\right] .
\end{aligned}
$$

Hence we have

$A_{1} \leq C\left[M_{T}^{2} \int_{\Omega} \lambda^{-2} \varphi^{2}\left(T^{\prime}\right) \mathrm{e}^{-2 s \eta\left(T^{\prime}\right)}|\xi|^{2} \mathrm{~d} x+s \int_{\omega_{T}} \varphi^{3}\left(|v|^{2}+\left|\partial_{t} v\right|^{2}\right) \mathrm{e}^{-2 s \eta} \mathrm{d} x \mathrm{~d} t\right]$.

Lower bounds for $A_{1^{-}}$We make the two following assumptions:

(H3a): $y\left(T^{\prime}\right)$ satisfies (HS3a),

(H3b): $\left(y\left(T^{\prime}\right), y^{\prime}\left(T^{\prime}\right)\right)$ satisfies (HS3b).

From (3.16) and (3.1) we have the following lower bound for $A_{1}$ : there exists $C(Q, \omega, c)>0$ such that

$$
\begin{aligned}
A_{1} \geq & C \delta^{2} s^{2} \lambda^{2} \int_{\Omega} \varphi^{3}\left(T^{\prime}\right) \mathrm{e}^{-2 s \eta\left(T^{\prime}\right)}|\xi|^{2}-C M_{1}^{2} s^{2} \lambda^{2} \int_{\omega} \varphi^{3}\left(T^{\prime}\right) \mathrm{e}^{-2 s \eta\left(T^{\prime}\right)}|\xi|^{2} \\
& -C(s, \lambda)\left\|\nabla\left(y\left(T^{\prime}, \cdot\right)-y^{\prime}\left(T^{\prime}, \cdot\right)\right)\right\|_{L^{\infty}(\Omega)},
\end{aligned}
$$

where $M_{1}$ is defined by (3.2), $\lambda>\lambda_{0}$ and $s>s_{0}\left(\lambda, \delta, M_{2}\right)$. From (3.18) and (3.19) we obtain

$$
\begin{aligned}
\delta^{2} s^{2} \lambda^{2} \int_{\Omega} \varphi^{3}\left(T^{\prime}\right) \mathrm{e}^{-2 s \eta}|\xi|^{2} \leq & C\left[M_{1}^{2} s^{2} \lambda^{2} \int_{\omega} \varphi^{3}\left(T^{\prime}\right) \mathrm{e}^{-2 s \eta}|\xi|^{2}+M_{T}^{2} \int_{\Omega} \lambda^{-2} \varphi\left(T^{\prime}\right)^{2} \mathrm{e}^{-2 s \eta}|\xi|^{2} \mathrm{~d} x\right. \\
& \left.+s \int_{\omega_{T}} \varphi^{3}|v|^{2} \mathrm{e}^{-2 s \eta} \mathrm{d} x \mathrm{~d} t+s^{-1} \int_{\omega_{T}} \varphi^{3}\left|\partial_{t} v\right|^{2} \mathrm{e}^{-2 s \eta} \mathrm{d} x \mathrm{~d} t\right] \\
& +C(s, \lambda)\left\|\nabla\left(y\left(T^{\prime}, \cdot\right)-y^{\prime}\left(T^{\prime}, \cdot\right)\right)\right\|_{L^{\infty}(\Omega)},
\end{aligned}
$$

if $\lambda>\lambda_{0}$ and $s>s_{0}\left(\lambda, \delta, M_{2}\right)$. So there exists $C>0$ such that

$$
\begin{array}{r}
\delta^{2} s^{2} \lambda^{2} \int_{\Omega} \varphi^{3}\left(T^{\prime}\right) \mathrm{e}^{-2 s \eta}|\xi|^{2} \leq C\left[M_{1}^{2} s^{2} \lambda^{2} \int_{\omega} \varphi^{3}\left(T^{\prime}\right) \mathrm{e}^{-2 s \eta}|\xi|^{2}+s \int_{\omega_{T}} \varphi^{3}|v|^{2} \mathrm{e}^{-2 s \eta} \mathrm{d} x \mathrm{~d} t\right. \\
\left.+s^{-1} \int_{\omega_{T}} \varphi^{3}\left|\partial_{t} v\right|^{2} \mathrm{e}^{-2 s \eta} \mathrm{d} x \mathrm{~d} t\right]+C(s, \lambda)\left\|\nabla\left(y\left(T^{\prime}, \cdot\right)-y^{\prime}\left(T^{\prime}, \cdot\right)\right)\right\|_{L^{\infty}(\Omega)},
\end{array}
$$

if $\lambda>\lambda_{0}$ and $s>s_{0}\left(\lambda, \delta, M_{T}, M_{2}, M_{T}\right)$.

Hence uniqueness for $c$ is proved since if $c=c^{\prime}$ in $\omega$ then the upper bound in (3.20) vanishes and so $\xi=0$ a.e in $\Omega$. In addition we have proved that (1.5) holds. 


\subsection{Checking the assumptions}

Let us show that we can check the assumptions (H3a), (H3b) and (H3c) if we make a sensible choice on $H$.

We recall the assumptions that $\Gamma$ is of class $\mathcal{C}^{2 n^{\prime}+2}$ and if $c \in \mathcal{E}$ then $S$ is of class $\mathcal{C}^{2 n^{\prime}+2}$ and $c_{\mid \Omega_{j}} \in \mathcal{C}^{2 n^{\prime}+1}\left(\overline{\Omega_{j}}\right), j=0,1$, where we set $n^{\prime}:=[n / 4]+1$.

We consider the following spatial self adjoint operator in $L^{2}(\Omega ; \mathrm{d} x)$ :

$$
A u:=-\operatorname{div}(c \nabla u), D(A):=\left\{u \in H_{0}^{1}(\Omega), A u \in L^{2}(\Omega)\right\} .
$$

Let us give some preliminary properties relating to $A$.

Firstly, we can easily check that for all $l \in \mathbb{N}, l \leq n^{\prime}$, if $j \in\{0,1\}$ and $p \in \mathcal{C}_{0}^{2 l+2}\left(\Omega_{j}\right)$, then $p \in D\left(A^{l+1}\right)$.

Secondly, if $0 \leq l \leq n^{\prime}$ and $p \in \mathcal{C}^{2 l+2}\left(\overline{\Omega_{j}}\right)$, then $\mathcal{A}^{l+1} p$ is defined in the classical sense and $\left(\mathcal{A}^{l} p\right)_{\mid \Omega_{j}} \in \mathcal{C}^{0}\left(\overline{\Omega_{j}}\right)$. Then we can claim

Proposition 3.1 Let $l \leq n^{\prime}, p \in L^{2}(\Omega)$ such that $p_{\mid \Omega_{j}} \in \mathcal{C}^{2 l+2}\left(\overline{\Omega_{j}}\right), p=\mathcal{A} p=\cdots=$ $\mathcal{A}^{l} p=0$ on $\Gamma,[p]=[\mathcal{A} p]=\cdots=\left[\mathcal{A}^{l} p\right]=0$ on $S,\left[c \partial_{n} p\right]=\left[c \partial_{n} \mathcal{A} p\right]=\cdots=\left[c \partial_{n} \mathcal{A}^{l} p\right]=0$ on $S$. Then $p \in D\left(A^{l+1}\right)$.

(A proof of proposition 3.1 is easily checked by induction). A sort of converse for this property is the following proposition, proved in [21, Corollary A.9] (see also [15, Proposition A.3, page 33]).

Proposition 3.2 Let $l \in \mathbb{N}$ and $c_{\mid \Omega_{j}} \in \mathcal{C}^{2 l+1}\left(\overline{\Omega_{j}}\right), j=0,1$, and $S$ and $\Gamma$ be of class $\mathcal{C}^{2 l+2}$. If $p \in D\left(A^{l+1}\right)$ then $p_{\mid \Omega_{j}} \in H^{2 l+2}\left(\Omega_{j}\right)$ and $\left\|p_{\mid \Omega_{j}}\right\|_{H^{2 l+2}\left(\Omega_{j}\right)} \leq C\|p\|_{D\left(A^{l+1}\right)}, j=0,1$.

Consequently if $l \leq n^{\prime}, r<2 l+2-\frac{1}{2} n$ and $p \in D\left(A^{l+1}\right)$ we then have, thanks to the Sobolev embeddings: $p_{\mid \Omega_{j}} \in \mathcal{C}^{r}\left(\overline{\Omega_{j}}\right)$ and

$$
\left\|p_{\mid \Omega_{j}}\right\|_{\mathcal{C}^{r}\left(\overline{\Omega_{j}}\right)} \leq C_{r, l}\|p\|_{D\left(A^{l+1}\right)}, j=0,1 .
$$

Since $n^{\prime}>\frac{n}{4}$, any value $r \leq 2$ satisfies the above condition if we set $l=n^{\prime}$.

Assumption (HS3a)- We check (HS3a) thanks to the two following results.

Lemma 3.1 Let $b \in \mathcal{C}^{0}(\bar{\Omega})$ such that $b_{\mid \Gamma}=0, b_{\mid \Omega_{i}} \in \mathcal{C}^{1}\left(\overline{\Omega_{i}}\right), i=0,1,[b]=0,\left[c \partial_{n} b\right]=0$. Then there exists a family of functions $b^{\varepsilon} \in D\left(A^{n^{\prime}+1}\right), \varepsilon>0$, which tends to $b$ for the strong topology of $W^{1, \infty}(\Omega)$ as $\varepsilon$ goes to 0 .

We prove lemma 3.1 in appendix.

Corollary 3.1 Let $b \in \mathcal{C}^{0}(\bar{\Omega})$ such that $b_{\mid \Gamma}=0, b_{\mid \Omega_{i}} \in \mathcal{C}^{1}\left(\overline{\Omega_{i}}\right), i=0,1,[b]=0$, $\left[c \partial_{n} b\right]=0$. Then for all $y_{0} \in L^{2}(\Omega)$ and $\varepsilon>0$ there exists a source term $H(t, x)$ vanishing for $0 \leq t \leq t_{0}$ and such that $\left\|Y\left(H, y_{0}\right)\left(T^{\prime}\right)-b\right\|_{W^{1, \infty}(\Omega)} \leq \varepsilon$.

\section{Proof}

Thanks to lemma (3.1) there exists $b^{\varepsilon} \in D\left(A^{n^{\prime}+1}\right)$ such that $\left\|b-b^{\varepsilon}\right\|_{W^{1, \infty}(\Omega)}<\frac{1}{2} \varepsilon$. The proof of [14, Proposition 1.1] holds for our operator $A$ and shows that for all $k \in \mathbb{N}$ and all $y_{0} \in L^{2}(\Omega)$ the set $\left\{Y\left(H, y_{0}\right)\left(T^{\prime}\right) ; H \in \mathcal{C}^{\infty}\left(\omega_{T}\right)\right\}$ is dense in $D\left(A^{k}\right)$ 
equipped with the norm $\|p\|_{D\left(A^{k}\right)}:=\left\|A^{k} p\right\|_{L^{2}(\Omega)}$. (Authors of [14] restrict their proof to the case $k=3$, but it is valid for any $k)$. So let $H \in \mathcal{C}^{\infty}\left(\omega_{T}\right)$ be such that $\left\|Y\left(H, y_{0}\right)\left(T^{\prime}\right)-b^{\varepsilon}\right\|_{D\left(A^{n^{\prime}+1}\right)}<\frac{1}{2}\left(C_{r, l}\right)^{-1} \varepsilon$ where $C_{r, l}>0$ is the constant in (3.21) with $r=1$. Hence

$\left\|Y\left(H, y_{0}\right)\left(T^{\prime}\right)-b\right\|_{W^{1, \infty}(\Omega)} \leq\left\|Y\left(H, y_{0}\right)\left(T^{\prime}\right)-b^{\varepsilon}\right\|_{W^{1, \infty}(\Omega)}+\left\|b-b^{\varepsilon}\right\|_{W^{1, \infty}(\Omega)} \leq \varepsilon$.

Now we check (H3a). Let $\varepsilon>0$. We recall that $\beta$ is constant on $\Gamma$. We apply Corollary 3.1 with $b=\beta-\beta_{\mid \Gamma}$. Hence there exists $H^{\varepsilon} \in \mathcal{C}_{0}^{\infty}\left(\omega_{T}\right)$ such that $\left\|\nabla Y\left(H^{\varepsilon}, y_{0}\right)\left(T^{\prime}\right)-\nabla \beta\right\|_{L^{\infty}(\Omega)}<\varepsilon$. Since there exists $\delta>0$ such that $|\nabla \beta(x)| \geq \delta>0$ for all $x \in \Omega \backslash \omega$, by choosing $\varepsilon=\delta / 2$ we check (H3a) (with $\delta$ replaced by $\delta^{2} / 2$ ).

Assumptions (H3b), (H3c) - We recall the following result (see [15, Proposition A.1, page 32]).

Proposition 3.3 Let $m \in \mathbb{N}$ and $c_{\mid \Omega_{i}} \in \mathcal{C}^{m+1}\left(\overline{\Omega_{i}}\right), i=0,1$, $S$ and $\Gamma$ of class $\mathcal{C}^{m+2}\left(\overline{\Omega_{i}}\right)$. For all $q_{0} \in L^{2}(\Omega)$ if we set $q=Y\left(H \equiv 0, q_{0}\right)$ then

$$
\left.\left.\left.\left.q \in \mathcal{C}\left([0, T] ; L^{2}(\Omega)\right) \cap \mathcal{C}^{1}(] 0, T\right] ; L^{2}(\Omega)\right) \cap \mathcal{C}(] 0, T\right] ; D(A)\right) .
$$

Furthermore $\left.\left.q \in \mathcal{C}^{k}(] 0, T\right], D\left(A^{l}\right)\right)$ for all $k, l \in \mathbb{N}$.

Similarly we have the following result.

Proposition 3.4 With the conditions and the notations of proposition 3.3, if we set $q=Y\left(H, q_{0}\right)$ with $q_{0} \in L^{2}(\Omega)$ and $H \in H^{2}\left(0, T ; D\left(A^{k}\right)\right)$ for some $k \in \mathbb{N}$, then

$$
\left.\left.q \in \mathcal{C}\left([0, T] ; H_{0}^{1}(\Omega)\right) \cap \mathcal{C}^{1}(] 0, T\right] ; H^{-1}(\Omega)\right) .
$$

Furthermore $\left.\left.\partial_{t}^{j} q \in \mathcal{C}^{0}(] 0, T\right], D\left(A^{k+\frac{1}{2}}\right)\right)$ for $j=0,1,2$.

We prove proposition 3.4 in appendix. Notice that if $H \in \mathcal{C}_{0}^{\infty}\left(\omega_{T}\right)$, then $H \in$ $H^{2}\left(0, T ; D\left(A^{n^{\prime}+1}\right)\right)$, thanks to proposition 3.1. Thus $y:=Y\left(H, y_{0}\right)$ satisfies $\partial_{t}^{j} y \in$ $\mathcal{C}^{0}\left(\left[t_{0}, T\right], D\left(A^{n^{\prime}+3 / 2}\right)\right)$. Since $n^{\prime}>n / 4$, Proposition 3.2 with $l=n^{\prime}$ and $r=2$ shows that $y$ satisfies (H3b). Since $n^{\prime}>n / 4-1 / 2$, Proposition 3.2 with $l=n^{\prime}$ and $r=1$ shows that $y$ satisfies (H3c). Since $c^{\prime} \in \mathcal{E}$ satisfies (iv) (see section 1.3), the same conclusion holds for $y^{\prime}=Y^{\prime}\left(H, y_{0}^{\prime}\right)$ : it satisfies (H3b) and (H3c) too.

\section{Inverse problem for case 2}

We prove (1.4). Weights $\varphi^{a} \mathrm{e}^{-b s \eta}$ of case 3 have to be replaced by $\sum_{i=1,2} \varphi_{i}^{a} \mathrm{e}^{-b s \eta_{i}}$.

\subsection{The stationary problem}

The main modification is due to the fact that assumption (HS3a) cannot simply be replaced by: " $\sum_{i=1,2}\left|\nabla \beta^{(i)} \nabla u\right| \geq \delta$ in $\Omega^{\prime \prime} \backslash \omega$ ", since this can not be checked. Thus we consider a couple of measurements $u_{1}, u_{2}$ satisfying the following assumptions 
Uniqueness of discontinuous diffusion coefficients for the heat equation

(HS2a) : $\left|\nabla u_{i} \cdot \nabla \beta^{(i)}\right| \geq \delta>0$ in $\Omega^{\prime \prime} \backslash\left(\omega \cup \omega_{i}\right), i=1,2$.

The result of this is that in $\Omega^{\prime \prime} \backslash \omega$ we have:

$$
\sum_{i=1,2}\left|\nabla u_{i} \cdot \nabla \beta^{(i)}\right| \geq \delta>0
$$

$(\mathrm{HS} 2 \mathrm{~b}): u_{i \mid \Omega_{j}} \in \mathcal{C}^{2}\left(\overline{\Omega_{j}}\right), u_{i \mid \Omega_{j}^{\prime}}^{\prime} \in \mathcal{C}^{2}\left(\overline{\Omega_{j}^{\prime}}\right), i=1,2, j=0,1$.

Then in this case theorem (3.1) becomes

Theorem 4.1 Let us give functions $f_{i} \in L^{2}(\Omega), u_{i} \in H^{1}(\Omega)$ (respectively $f_{i}^{\prime} \in L^{2}(\Omega)$, $u_{i}^{\prime} \in H^{1}(\Omega)$ ), $i=1,2$, and a solution $c \in \mathcal{E}$ (respectively $c^{\prime} \in \mathcal{E}$ ) of the following first-order partial differential equations

$$
-\operatorname{div}\left(c \nabla u_{i}\right)=f_{i}\left(\text { respectively }-\operatorname{div}\left(c^{\prime} \nabla u_{i}^{\prime}\right)=f_{i}^{\prime}\right) \text { in } \Omega, i=1,2 .
$$

If assumptions (HS2a) and (HS2b) are checked then we have:

$$
\begin{aligned}
\delta^{2} \int_{\Omega} \sum_{i=1,2} \varphi_{i}^{3}\left(T^{\prime}\right) \mathrm{e}^{-2 s \eta_{i}\left(T^{\prime}\right)}|\xi|^{2} \mathrm{~d} x \leq C s^{-2} \lambda^{-2} \int_{\Omega} \sum_{i=1,2} \varphi_{i}\left(T^{\prime}\right) \mathrm{e}^{-2 s \eta_{i}}\left|f_{i}-f_{i}^{\prime}\right|^{2} \\
+C M_{1}^{2} \int_{\omega} \sum_{i=1,2} \varphi_{i}^{3} \mathrm{e}^{-2 s \eta_{i}}|\xi|^{2} \mathrm{~d} x+C(s, \lambda) \sum_{i=1,2}\left\|\nabla\left(u_{i}-u_{i}^{\prime}\right)\right\|_{L^{\infty}(\Omega)},
\end{aligned}
$$

if $\lambda \geq \lambda_{0}$ and $s \geq s_{0}\left(\lambda, \delta, M_{2}\right)$, where we set

$$
M_{j}:=\max \left(\max _{i=1,2}\left(\left\|u_{i}\right\|_{W^{j, \infty}\left(\Omega^{\prime \prime}\right)},\left\|u_{i}^{\prime}\right\|_{W^{j, \infty}\left(\Omega^{\prime \prime}\right)}\right)\right), j=1,2 .
$$

\section{Proof}

We give a sketch of proof. Details of computations and estimates are similar to case 3 .

If $q$ is a function in $\Omega$, we denote $q_{s, i} \equiv \varphi_{i}^{\frac{1}{2}} \mathrm{e}^{-s \eta_{i}} q, i=1,2$.

We set for $i=1,2$ :

$$
\begin{aligned}
P_{i} u_{i} & :=-\operatorname{div}\left(c_{s, i} \nabla u_{i}\right)-s \nabla \eta_{i} c_{s, i} \cdot \nabla u_{i}=f_{s, i}-\frac{1}{2} c_{s, i} \frac{\nabla \varphi_{i}}{\varphi_{i}} \cdot \nabla u_{i}, \\
P_{i}^{\prime} u_{i}^{\prime} & :=-\operatorname{div}\left(c_{s, i}^{\prime} \nabla u_{i}^{\prime}\right)-s \nabla \eta_{i} c_{s, i}^{\prime} \cdot \nabla u_{i}^{\prime}=f_{s, i}^{\prime}-\frac{1}{2} c_{s, i}^{\prime} \frac{\nabla \varphi_{i}}{\varphi_{i}} \cdot \nabla u_{i}^{\prime}, \\
M_{s, i} & :=P_{i} u_{i}-P_{i}^{\prime} u_{i}^{\prime}, \\
I_{i}\left(u_{i}, u_{i}^{\prime}\right) & :=\left\|M_{s, i}\right\|_{L^{2}(\Omega ; c(x) \mathrm{d} x)}^{2} \\
B_{1, i} & :=\int_{\Omega^{\prime \prime}}\left|\nabla \eta_{i} \cdot\left(c_{s, i} \nabla u_{i}-c_{s, i}^{\prime} \nabla u_{i}^{\prime}\right)\right|^{2} c(x) \mathrm{d} x, \\
B_{2, i} & :=\int_{\Omega^{\prime \prime}}\left(\operatorname{div}\left(c_{s, i}^{\prime} \nabla u_{i}^{\prime}\right)-\operatorname{div}\left(c_{s, i} \nabla u_{i}\right)\right) \nabla \eta_{i} \cdot\left(c_{s, i}^{\prime} \nabla u_{i}^{\prime}-c_{s, i} \nabla u_{i}\right) c(x) \mathrm{d} x .
\end{aligned}
$$

Then we set

$$
\begin{aligned}
I^{(2)} & :=\sum_{i=1,2} I_{i}\left(u_{i}, u_{i}^{\prime}\right), \\
B_{j}^{(2)} & :=\sum_{i=1,2} B_{j, i}, j=1,2,
\end{aligned}
$$

which respectively replace $I\left(u, u^{\prime}\right)$ and $B_{j}$ of case 3 . Thanks to (HS2a), $B_{1}^{(2)}$ satisfies: $B_{1}^{(2)} \geq \frac{1}{2} c_{\min } \delta^{2} \lambda^{2} \int_{\Omega \backslash \omega} \sum_{i=1,2} \varphi_{i}^{2}\left|\xi_{s, i}\right|^{2} \mathrm{~d} x-C \lambda^{2} \int_{\Omega} \sum_{i=1,2} \varphi_{i}^{3} \mathrm{e}^{-2 s \eta_{i}}\left|\nabla\left(u_{i}-u_{i}^{\prime}\right)\right|^{2} \mathrm{~d} x$ 
Uniqueness of discontinuous diffusion coefficients for the heat equation

Thanks to (HS2b) we have for $i=1,2$ :

$$
B_{2, i} \geq-C\left(\lambda^{2} M_{1}^{2}+\lambda M_{2}^{2}\right) \int_{\Omega^{\prime \prime}} \varphi_{i}\left|\xi_{s, i}\right|^{2}-C(s, \lambda) M_{2}\left\|\nabla\left(u_{i}-u_{i}^{\prime}\right)\right\|_{L^{\infty}(\Omega)} .
$$

Then we obtain

$$
\begin{aligned}
\sum_{i=1,2}\left\{C\left(\left\|f_{s, i}-f_{s, i}^{\prime}\right\|^{2}+\lambda^{2} M_{1}^{2} \int_{\Omega}\left|\xi_{s, i}\right|^{2}\right)+C(s, \lambda)\left\|\nabla\left(u_{i}-u_{i}^{\prime}\right)\right\|_{L^{\infty}(\Omega)}^{2}\right\} \\
\geq I^{(2)} \geq \sum_{i=1,2}\left\{C \delta^{2} s^{2} \lambda^{2} \int_{\Omega \backslash \omega} \varphi_{i}^{2}\left|\xi_{s, i}\right|^{2} \mathrm{~d} x-C M_{1}^{2} s^{2} \lambda^{2} \int_{\omega} \varphi_{i}^{2}\left|\xi_{s, i}\right|^{2} \mathrm{~d} x\right. \\
\left.\quad-C\left(\lambda^{2} M_{1}^{2}+\lambda M_{2}^{2}\right) s \int_{\Omega} \varphi_{i}\left|\xi_{s, i}\right|^{2}-C(s, \lambda) M_{2}\left\|\nabla\left(u_{i}-u_{i}^{\prime}\right)\right\|_{L^{\infty}(\Omega)}\right\} .
\end{aligned}
$$

Then we can conclude.

\subsection{The non stationary problem}

We consider two solutions $y_{i}$ (respectively $y_{i}^{\prime}$ ) of the equations for $c$ (respectively $c^{\prime}$ ). We set $v_{i}=\partial_{t}\left(y_{i}-y_{i}^{\prime}\right), u_{i}=y_{i}\left(T^{\prime}, \cdot\right)$. Assumption $(\mathrm{H} 3 \mathrm{c})$ is replaced by

(H2c): $\nabla \partial_{t}^{k} y_{i}^{\prime} \in L^{2}\left(t_{0}, T ;\left(L^{\infty}(\Omega)\right)^{n}\right), i, k=1,2$.

We set

$$
\begin{aligned}
M_{T, k} & :=\max _{i, k=1,2}\left\|\nabla \partial_{t}^{k} y_{i}^{\prime}\right\|_{L^{2}\left(t_{0}, T ;\left(L^{\infty}(\Omega)\right)^{n}\right)}, \\
M_{T} & :=\max _{k=1,2} M_{T, k} .
\end{aligned}
$$

Furthermore we make the two following assumptions:

(H2a): $\left(y_{i}\left(T^{\prime}\right) ; i=1,2\right)$ satisfies (HS2a),

(H2b): $\left(y_{i}\left(T^{\prime}\right), y_{i}^{\prime}\left(T^{\prime}\right) ; i=1,2\right)$ satisfies (HS2b).

We set $A_{1, i, j}:=\int_{\Omega} \frac{1}{2}\left|v_{i}\left(T^{\prime}\right)\right|^{2} \varphi_{j}\left(T^{\prime}\right) \mathrm{e}^{-2 s \eta_{j}\left(T^{\prime}\right)}, i, j=1,2$. An upper bound for $A_{1, i, j}$ is obtained by following the way to estimate $A_{1}$ of case 3 (see (3.18)). We then obtain

$$
\begin{aligned}
A_{1, i, j} \leq & C\left(M_{T}^{2} \int_{\Omega} \sum_{k=1,2} \lambda^{-2} \varphi_{k}^{2}\left(T^{\prime}\right) \mathrm{e}^{-2 s \eta_{k}\left(T^{\prime}\right)}|\xi|^{2}+\right. \\
& \left.+s \int_{\omega_{T}} \sum_{k=1,2} \varphi_{k}^{3} \mathrm{e}^{-2 s \eta_{k}}\left(\left|v_{i}\right|^{2}+\left|\partial_{t} v_{i}\right|^{2}\right) \mathrm{d} x \mathrm{~d} t\right), i, j=1,2 .
\end{aligned}
$$

On the other hand, estimate (3.19) is replaced by:

$$
\begin{aligned}
\sum_{i=1,2} A_{1, i, i} \geq & C \delta^{2} \int_{\Omega} s^{2} \lambda^{2} \sum_{j=1,2} \varphi_{j}^{3}\left(T^{\prime}\right) \mathrm{e}^{-2 s \eta_{j}\left(T^{\prime}\right)}|\xi|^{2}-C M_{1}^{2} s^{2} \lambda^{2} \int_{\omega} \sum_{j=1,2} \varphi_{j}^{3}\left(T^{\prime}\right) \mathrm{e}^{-2 s \eta_{j}\left(T^{\prime}\right)}|\xi|^{2} \\
& -C(s, \lambda) \sum_{i=1,2}\left\|\nabla\left(y_{i}\left(T^{\prime}, \cdot\right)-y_{i}^{\prime}\left(T^{\prime}, \cdot\right)\right)\right\|_{L^{\infty}(\Omega)}
\end{aligned}
$$

Hence we obtain:

$\delta^{2} \int_{\Omega} \sum_{i=1,2} s^{2} \lambda^{2} \varphi_{i}^{3}\left(T^{\prime}\right) \mathrm{e}^{-2 s \eta_{i}}|\xi|^{2} \leq C\left(M_{1}^{2} s^{2} \lambda^{2} \int_{\omega} \sum_{i=1,2} \varphi_{i}^{3}\left(T^{\prime}\right) \mathrm{e}^{-2 s \eta_{i}}|\xi|^{2}\right.$ 


$$
\begin{aligned}
& \left.+\int_{\omega_{T}} \sum_{i, j=1,2} s \varphi_{i}^{3} \mathrm{e}^{-2 s \eta_{i}}\left(\left|v_{j}\right|^{2}+\left|\partial_{t} v_{j}\right|^{2}\right) \mathrm{d} x \mathrm{~d} t\right) \\
& +C(s, \lambda) \sum_{i=1,2}\left\|\nabla\left(y_{i}\left(T^{\prime}, \cdot\right)-y_{i}^{\prime}\left(T^{\prime}, \cdot\right)\right)\right\|_{L^{\infty}(\Omega)},
\end{aligned}
$$

if $\lambda>\lambda_{0}$ and $s>s_{0}\left(\lambda, \delta, M_{2}, M_{T}\right)$. Hence (1.4).

\subsection{Checking the assumptions}

This is identical to case 3 .

\section{Inverse problem for case 1}

By modifying the results of case 2 as follows, we can obtain (1.2). We reduce to the case 2 as already described: we extend $\Omega$ near a relative open subset of $\gamma$ to an connected open domain $\tilde{\Omega}$. This leads to the construction of geometrical functions $\beta^{(i)} \in \mathcal{C}^{0}(\tilde{\Omega})$, $i=1,2$ (see [15]) by reduction to case 2 with $\tilde{\Omega}$ instead of $\Omega$. Then, except for the Carleman estimate with data in $H^{-1}$ which we prove in appendix, computations are similar to case 2 by formally setting $\omega=\emptyset$ and by keepping integrals on $\gamma$ in the leading terms.

\subsection{The stationary problem}

As for case 2 we make the following assumptions

(HS1a): $\left|\nabla u_{i} \cdot \nabla \beta^{(i)}\right| \geq \delta>0$ in $\Omega^{\prime \prime} \backslash \omega_{i}, i=1,2$.

The result is that in $\Omega^{\prime \prime}$ we have:

$$
\sum_{i=1,2}\left|\nabla u_{i} \cdot \nabla \beta^{(i)}\right| \geq \delta>0 \text { in } \Omega^{\prime \prime},
$$

even if it means to change $\delta$ (we keep the same notation).

(HS1b): $u_{i \mid \Omega_{j}} \in \mathcal{C}^{2}\left(\overline{\Omega_{j}}\right), u_{i \mid \Omega_{j}^{\prime}}^{\prime} \in \mathcal{C}^{2}\left(\overline{\Omega_{j}^{\prime}}\right), i=1,2, j=0,1$.

Constants $M_{j}$ are defined by (4.2). Theorem 4.1 becomes the following one with similar notations:

Theorem 5.1 Under assumptions (HS1a) and (HS1b), we have the estimate

$$
\begin{aligned}
& \delta^{2} \int_{\Omega} \sum_{i=1,2} \varphi_{i}^{3}\left(T^{\prime}\right) \mathrm{e}^{-2 s \eta_{i}\left(T^{\prime}\right)}|\xi|^{2} \mathrm{~d} x \leq C s^{-2} \lambda^{-2} \int_{\Omega} \sum_{i=1,2} \varphi_{i}\left(T^{\prime}\right) \mathrm{e}^{-2 s \eta_{i}\left(T^{\prime}\right)}\left|f_{i}-f_{i}^{\prime}\right|^{2} \\
& +C M_{1}^{2} \int_{\gamma} \sum_{i=1,2} s^{-1} \lambda^{-1} \varphi_{i}^{2}\left(T^{\prime}\right) \mathrm{e}^{-2 s \eta_{j}\left(T^{\prime}\right)} \xi^{2}+C(s, \lambda) \sum_{i=1,2}\left\|\nabla\left(u_{i}-u_{i}^{\prime}\right)\right\|_{L^{\infty}(\Omega)},
\end{aligned}
$$

if $\lambda \geq \lambda_{0}$ and $s \geq s_{0}\left(\lambda, \delta, M_{2}\right)$. 
Uniqueness of discontinuous diffusion coefficients for the heat equation

Computations and estimates for case 1 straightforwardly follow those of case 2 , with the only difference that we have to take care of the integrals on $\Gamma$ which correspond to $B_{3}$ (and to $B_{71}=-B_{3}$ ) of case 3 . Thus we set (compare with (3.13)):

$$
B_{3}^{(1)}:=\int_{\Gamma} \sum_{i=1,2} \varphi_{i} \mathrm{e}^{-2 s \eta_{i}} \xi^{2}\left(c \partial_{n} \eta_{i}\right)\left(\partial_{n} u_{i}\right)^{2} \mathrm{~d} x
$$

Thanks to the properties of $\beta^{(i)}$ we then have

$$
\begin{aligned}
B_{3}^{(1)} & =-\int_{\Gamma} \sum_{i=1,2} \lambda \varphi_{i}^{2} \mathrm{e}^{-2 s \eta_{i}} \partial_{n} \beta^{(i)}\left(\partial_{n} u_{i}\right)^{2}|\xi|^{2} c(x) \mathrm{d} x \\
& \geq-M_{1}^{2} \int_{\gamma_{i=1,2}} \lambda \varphi_{i}^{2} \mathrm{e}^{-2 s \eta_{j}} \xi^{2} \mathrm{~d} \sigma(x) .
\end{aligned}
$$

Finally, we notice that the integral correponding to $B_{71}$ equals $-B_{3}^{(1)}$.

\subsection{The non stationary problem}

We consider the solutions $y_{i}=Y\left(h_{i}, y_{0}\right), y_{i}^{\prime}=Y^{\prime}\left(h_{i}, y_{0}^{\prime}\right), i=1,2$, of the equations. We set $v_{i}=\partial_{t}\left(y_{i}-y_{i}^{\prime}\right), u_{i}=y_{i}\left(T^{\prime}, \cdot\right)$. We make the following assumption

(H1c): $\nabla \partial_{t}^{k} y_{i}^{\prime} \in L^{2}\left(t_{0}, T ;\left(L^{\infty}(\Omega)\right)^{n}\right), i, k=1,2$.

(Formally, this is (H2c)). Constant $M_{T}$ is defined by (4.3). In addition we make the two following assumptions:

(H1a): $\left(y_{i}\left(T^{\prime}\right) ; i=1,2\right)$ satisfies (HS1a),

(H1b): $\left(y_{i}\left(T^{\prime}\right), y_{i}^{\prime}\left(T^{\prime}\right) ; i=1,2\right)$ satisfies (HS1b).

Integral $A_{1, i, j}$ and estimate (4.4) are replaced by

$$
\begin{aligned}
A_{1, i, j}:= & \frac{1}{2} \int_{\Omega}\left|v_{i}\left(T^{\prime}\right)\right|^{2} \varphi_{j}\left(T^{\prime}\right) \mathrm{e}^{-2 s \eta_{j}\left(T^{\prime}\right)} \\
\leq & C M_{T}^{2} \int_{\Omega} \sum_{k} \lambda^{-2} \varphi_{k}^{2}\left(T^{\prime}\right) \mathrm{e}^{-2 s \eta_{k}\left(T^{\prime}\right)}|\xi|^{2} \mathrm{~d} x+C \int_{\gamma} \sum_{k} s^{-1} \lambda^{-3} \varphi_{k}\left(T^{\prime}\right) \mathrm{e}^{-2 s \eta_{k}\left(T^{\prime}\right)}|\xi|^{2} \mathrm{~d} \sigma(x) \\
& +C \int_{\gamma_{T}} \sum_{k} s^{-1} \lambda^{-3} \varphi_{k} \mathrm{e}^{-2 s \eta_{k}}\left(\left|\partial_{n} v_{i}\right|^{2}+C s^{-2}\left|\partial_{n} \partial_{t} v_{i}\right|^{2}\right) \mathrm{d} \sigma(x) \mathrm{d} t, i, j=1,2 .
\end{aligned}
$$

Estimate (4.5) is replaced by:

$$
\begin{aligned}
\sum_{i=1,2} A_{1, i, i} \geq & C\left(\delta^{2} \int_{\Omega} \sum_{i=1,2} s^{2} \lambda^{2} \varphi_{i}^{3}\left(T^{\prime}\right) \mathrm{e}^{-2 s \eta_{i}\left(T^{\prime}\right)}|\xi|^{2}-M_{1}^{2} \int_{\gamma_{i=1,2}} s \lambda \varphi_{i}^{2}\left(T^{\prime}\right) \mathrm{e}^{-2 s \eta_{i}\left(T^{\prime}\right)}|\xi|^{2}\right) \\
& -C(s, \lambda) \sum_{i=1,2}\left\|\nabla\left(y_{i}\left(T^{\prime}, \cdot\right)-y_{i}^{\prime}\left(T^{\prime}, \cdot\right)\right)\right\|_{L^{\infty}(\Omega)} .
\end{aligned}
$$

Finally we obtain:

$$
\begin{gathered}
\delta^{2} \int_{\Omega} \sum_{i=1,2} s^{2} \lambda^{2} \varphi_{i}^{3}\left(T^{\prime}\right) \mathrm{e}^{-2 s \eta_{i}\left(T^{\prime}\right)}|\xi|^{2} \leq C M_{1}^{2} \int_{\gamma} \sum_{i=1,2} s \lambda \varphi_{i}^{2}\left(T^{\prime}\right) \mathrm{e}^{-2 s \eta_{i}\left(T^{\prime}\right)}|\xi|^{2} \mathrm{~d} \sigma(x) \mathrm{d} t \\
+C \int_{\gamma_{T}} \sum_{i, j, k=1,2} s^{-1} \lambda^{-3} \varphi_{i}^{3} \mathrm{e}^{-2 s \eta_{i}}\left|\partial_{t}^{k} \partial_{n}\left(y_{j}-y_{j}^{\prime}\right)\right|^{2} \mathrm{~d} \sigma(x) \mathrm{d} t
\end{gathered}
$$


Uniqueness of discontinuous diffusion coefficients for the heat equation

$$
+C(s, \lambda) \sum_{i=1,2}\left\|\nabla\left(y_{i}\left(T^{\prime}, \cdot\right)-y_{i}^{\prime}\left(T^{\prime}, \cdot\right)\right)\right\|_{L^{\infty}(\Omega)},
$$

if $\lambda>\lambda_{0}$ and $s>s_{0}\left(\lambda, \delta, M_{2}, M_{T}\right)$.

\subsection{Checking assumptions}

Thanks to an extension of $\Omega$ by $\hat{\Omega}$, we straightforwardly come back to case 2 . For example, let us check (H1a). We extend any initial data $y_{0} \in L^{2}(\Omega)$ by 0 in $\hat{\Omega} \backslash \Omega$. Then there exist two source terms $H^{(i)} \in \mathcal{C}_{0}^{\infty}\left(\omega_{T}\right)$ such that the trajectory $\hat{Y}\left(H^{(i)}, y_{0}\right)$ solution of:

$$
\left\{\begin{array}{l}
\mathcal{L} y=H^{(i)} \chi_{\omega} \text { in }(0, T) \times \hat{\Omega}, \\
y(t, \cdot)=0 \text { on } \partial \hat{\Omega}, \\
\hat{y}(0, x)=y_{0}(x) \text { in } \hat{\Omega},
\end{array}\right.
$$

satisfies $\nabla \hat{Y}\left(H^{(i)}, y_{0}\right)\left(T^{\prime}\right) \cdot \nabla \beta^{(i)} \geq \delta>0$ in $\hat{\Omega} \backslash\left(\omega \cup \omega_{i}\right)$. Let us set $h^{(i)}:=\hat{Y}\left(H^{(i)}, y_{0}\right)$ on $\Sigma_{0}$. Then the function $y^{(i)}:=\hat{Y}\left(H^{(i)}, y_{0}\right)_{\mid Q}$ is solution of:

$$
\begin{cases}\mathcal{L} y^{(i)} & =0 \text { in } Q_{0}, \\ y^{(i)}(t, \cdot) & =h^{(i)} \text { on } \Gamma, \\ y^{(i)}(0, x) & =y_{0}(x) \text { in } \Omega,\end{cases}
$$

and satisfies $\nabla y^{(i)}\left(T^{\prime}, \cdot\right) \cdot \nabla \beta^{(i)} \geq \delta>0$ in $\Omega \backslash \omega_{i}$.

\section{Appendix}

We denote the formal heat operator by $\mathcal{L} q:=\partial_{t} q+\mathcal{A} q$, with $\mathcal{A}:=-\operatorname{div}(c \nabla \cdot)$, and its formal adjoint by $\mathcal{L}^{*}:=-\partial_{t}+\mathcal{A}$. We set $D(A)=\left\{u \in H_{0}^{1}(\Omega) ; \mathcal{A} u \in L^{2}(\Omega)\right\}$ and $D\left(L^{(*)}\right):=\left\{z: z \in L^{2}\left(t_{0}, T ; H_{0}^{1}(\Omega)\right), \mathcal{L}^{(*)} z \in L^{2}(Q)\right\}$ (see also remark 2.1).

\subsection{Proof of Estimate (2.4)}

In this section we explain how to obtain the Carleman estimate (2.4) in the case $q \in D(L)$. In the same way we obtain (2.4) for $q \in D\left(L^{*}\right)$ and (2.6), (2.8) for $q \in D\left(L^{(*)}\right)$. We set

$$
\begin{aligned}
Z_{0}:= & \left\{q: q_{\mid \Omega_{j}} \in \mathcal{C}^{2}\left(\left[t_{0}, T\right] \times \overline{\Omega_{j}}\right), j=0,1,[q]=0,\left[c \partial_{n} q\right]=0, q=0 \text { on } \Sigma\right\}, \\
Z:= & \left\{q \in L^{2}(Q): q_{\mid \Omega_{j}} \in L^{2}\left(t_{0}, T ; H^{2}\left(\Omega_{j}\right)\right), j=0,1, \partial_{t} q \in L^{2}\left(t_{0}, T ; H^{1}(\Omega)\right),\right. \\
& {\left.[q]=0 \text { p.p in }\left(t_{0}, T\right) \times S,\left[c \partial_{n} q\right]=0 \text { p.p in }\left(t_{0}, T\right) \times S, q=0 \text { on } \Sigma\right\} . }
\end{aligned}
$$

Authors of [17] prove (2.4) for all $q \in Z_{0}$. We notice that their proof directly holds for any $q \in Z$ (for example, they obtain integrals on $S$ that keep sense if $q \in Z$ since in this case $\left(\nabla\left(q_{\mid \Omega_{j}}\right)\right)_{\mid S}$ belongs to $\left.\left(L^{2}\left(t_{0}, T ; H^{\frac{1}{2}}(S)\right)\right)^{n}\right)$.

Let $q \in D(L), q_{0}:=q\left(t_{0}\right) \in L^{2}(\Omega)$ and $f:=L q \in L^{2}(Q)$. Then $q$ is the unique function 
in $L^{2}\left(t_{0}, T ; H_{0}^{1}(\Omega)\right)$ satisfying $L q=f$ and $q\left(t_{0}, \cdot\right)=q_{0}$. We refer to [22, Theorems 1.1, 4.1 and Remark 4.3, Chap. 3] for a complete proof. Thus $q \in \mathcal{C}^{0}\left(\left[t_{0}, T\right], L^{2}(\Omega)\right)$ and

$$
\|q\|_{L^{2}\left(t_{0}, T ; H_{0}^{1}(\Omega)\right)} \leq C\left(\|f\|_{L^{2}(Q)}+\left\|q_{0}\right\|_{L^{2}(\Omega)}\right) .
$$

Let $\left(e_{j}\right)_{j \geq 0}$ be the orthonormal familly of eigenvectors of $A$ associated with the non decreasing familly of eigenvalues $\left(\lambda_{j}\right)_{j \geq 0}$. For $\varepsilon>0$ we set

$$
q_{0, \varepsilon}:=\sum_{0 \leq j \leq 1 / \varepsilon}\left(q_{0} \mid e_{j}\right) e_{j} .
$$

Thus $q_{0, \varepsilon} \in D\left(A^{r}\right)$ for all $r \in \mathbb{N}$ and $q_{0, \varepsilon} \rightarrow q\left(t_{0}, \cdot\right)$ in $L^{2}(Q)$ (in the strong sense) as $\varepsilon$ goes to 0 .

We then prove the existence of a familly $\left(f_{\varepsilon}\right)_{\varepsilon>0}$ of functions such that $f_{\varepsilon} \in$ $\mathcal{C}^{k}\left(\left[t_{0}, T\right], D\left(A^{r}\right)\right)$ for all $k, r \in \mathbb{N}$, and $f_{\varepsilon} \rightarrow f$ in $L^{2}(Q)$ (in the strong sense) as $\varepsilon$ goes to 0 . Let $f_{j}(t):=\left(f(t, \cdot) \mid e_{j}\right)$ and $R_{\varepsilon}$ be a smoothing mapping from $L^{2}\left(t_{0}, T\right)$ into $\mathcal{C}^{\infty}\left(\left[t_{0}, T\right]\right)$. Thus the familly of functions $f_{\varepsilon}=\sum_{0 \leq j \leq 1 / \varepsilon}\left(R_{\varepsilon} f_{j}(t) \mid e_{j}\right) e_{j}$ satisfies the above conditions.

Let $q_{\varepsilon} \in D(L)$ be the solution of $L q_{\varepsilon}=f_{\varepsilon}$ in $Q$ and $q_{\varepsilon}\left(t_{0}, \cdot\right)=q_{0, \varepsilon}$. We have

$$
q_{\varepsilon}(t, \cdot)=e^{\left(t_{0}-t\right) A} q_{0, \varepsilon}+\int_{t_{0}}^{t} e^{(s-t) A} f_{\varepsilon}(s) d s .
$$

From the spectral representation of $A$ we thus have $q_{\varepsilon} \in \mathcal{C}^{k}\left(\left[t_{0}, T\right], D\left(A^{r}\right)\right)$ for all $k, r \in \mathbb{N}$. Thanks to Proposition 3.2 with $r=1$, we obtain $q_{\varepsilon} \in Z$.

We apply (6.1) with $\left(f, q_{0}, q\right)$ replaced by $\left(f-f_{\varepsilon}, q_{0}-q_{0, \varepsilon}, q-q_{\varepsilon}\right)$. This shows that $q_{\varepsilon}$ tends to $q$ in $L^{2}\left(t_{0}, T ; H^{1}(\Omega)\right)$ as $\varepsilon$ goes to 0 . Since the weights $\Pi_{j}$ are bounded in $Q$, for all $j$ (in all cases $1,2,3$ ), then $\left(\Pi_{1}\right)^{\frac{1}{2}} q_{\varepsilon}$ tends to $\left(\Pi_{1}\right)^{\frac{1}{2}} q$ in $L^{2}(Q)$ and $\left(s \lambda^{2} \varphi e^{-2 s \eta}\right)^{\frac{1}{2}} \nabla q_{\varepsilon}$ strongly tends to $\left(s \lambda^{2} \varphi e^{-2 s \eta}\right)^{\frac{1}{2}} \nabla q$ in $\left(L^{2}(Q)\right)^{n}$. Finally, we get the conclusion since $\left(\Pi_{2}\right)^{\frac{1}{2}} f_{\varepsilon}$ tends to $\left(\Pi_{2}\right)^{\frac{1}{2}} f$ in $L^{2}(Q)$ and since $(2.4)$ holds for $q_{\varepsilon}$.

\subsection{Proof of Estimates (2.5), (2.7), (2.9)}

We consider the following formal equations with data in $H^{-1}$ :

$$
\left\{\begin{array}{l}
\mathcal{L} q=\operatorname{div} f \text { in } Q=\left(t_{0}, T\right) \times \Omega, \\
q=0 \text { on } \Sigma=\left(t_{0}, T\right) \times \partial \Omega, \\
q\left(t_{0}\right)=q_{0} \text { in } \Omega .
\end{array}\right.
$$

By definition, the function $q$ is solution of $(6.2)$ in $L^{2}(Q)$ if and only if it satisfies for any $g \in L^{2}(Q)$ :

$$
\int_{Q} q g \mathrm{~d} x \mathrm{~d} t=-\int_{Q} f \cdot \nabla z \mathrm{~d} x \mathrm{~d} t+\int_{\Omega} q_{0} z\left(t_{0}\right) \mathrm{d} x,
$$

where $z \in D\left(L^{*}\right)$ is solution of $L^{*} z=g, z(T)=0$.

Remark 6.1 If $f \in\left(L^{2}(Q)\right)^{n}$ then divf $\in L^{2}\left(t_{0}, T ; H^{-1}(\Omega)\right)$ and so the problem (6.2) with data $q_{0} \in L^{2}(\Omega)$ admits an unique solution $q \in L^{2}\left(t_{0}, T ; H_{0}^{1}(\Omega)\right)$. Moreover we have $q \in \mathcal{C}^{0}\left(\left[t_{0}, T\right], L^{2}(\Omega)\right)$ and $\partial_{t} q \in L^{2}\left(t_{0}, T ; H^{-1}(\Omega)\right)$. We refer to [22, Theorems 1.1, 4.1 and Remark 4.3, Chap 3] for a complete proof. 
Proof for Case 3 We prove that (2.5) holds for case 3. We set

$$
\Pi_{1}(t, x):=s^{3} \lambda^{4} \varphi^{3} \mathrm{e}^{-2 s \eta}, \Pi_{2}(t, x):=\mathrm{e}^{-2 s \eta}, \Pi_{3}(t, x):=s^{2} \lambda^{2} \varphi^{2} \mathrm{e}^{-2 s \eta} .
$$

We solve the following equation with unknown $p \in P_{0}:=\mathcal{C}^{2}\left(\left[t_{0}, T\right] ; D\left(A^{2}\right)\right)$ :

$$
\mathcal{L}^{*}\left(\Pi_{2} \mathcal{L} p\right)+\Pi_{1} \chi_{\omega} p=\Pi_{1} q \text { in } Q,
$$

where we fix $q \in L^{2}(Q)$, solution of (6.2). We define on $\left(P_{0}\right)^{2}$ the bilinear symmetric form $a(\cdot, \cdot)$ and on $P_{0}$ the linear form $b(\cdot)$ as follows :

$$
\begin{aligned}
a\left(p, p^{\prime}\right) & :=\int_{Q} \Pi_{2} \mathcal{L} p \mathcal{L} p^{\prime}+\int_{\omega_{T}} \Pi_{1} p p^{\prime}, \\
b_{q}\left(p^{\prime}\right) & :=\int_{Q} \Pi_{1} q p^{\prime} .
\end{aligned}
$$

Note that $P_{0} \subset D(L)$ and so we can apply the Carleman estimate (2.4):

$$
\int_{Q} \Pi_{1} p^{2} \leq C(Q, \omega, c) a(p, p),
$$

for all $p \in P_{0}$. This implies that $P_{0}$ equipped with $\|p\|_{a}:=\sqrt{a(p, p)}$ is a normed space. Then we denote by $P$ the closure of $P_{0}$ with this norm. So $P$ equipped with the bilinear form $a(\cdot, \cdot)$ is an Hilbert space and (6.6) holds for any $p \in P$. Thanks to the Cauchy-Schwarz estimate and to (6.6) we then have for any $p \in P$ :

$$
\left|b_{q}(p)\right| \leq\left(\int_{Q} \Pi_{1}|q|^{2}\right)^{\frac{1}{2}}\left(\int_{Q} \Pi_{1}|p|^{2}\right)^{\frac{1}{2}} \leq C^{\frac{1}{2}}(Q, \omega, c)\left(\int_{Q} \Pi_{1}|q|^{2}\right)^{\frac{1}{2}}\|p\|_{a} .
$$

So the form $b_{q}$ is $a$-continuous. Hence we can apply the Lax-Milgram theorem to the following equation with unknown $p \in P$ :

$$
a\left(p, p^{\prime}\right)=b_{q}\left(p^{\prime}\right) \text { for any } p^{\prime} \in P .
$$

We denote by $p$ the unique solution in $P$ of (6.8), and we set $u:=-\Pi_{1} \chi_{\omega} p, g=u+\Pi_{1} q$, $z=\Pi_{2} \mathcal{L} p$. Notice that, thanks to (6.5) and (6.11), we have $z \in D\left(L^{*}\right), L^{*} z=g=$ $u+\Pi_{1} q, z\left(t_{0}, \cdot\right)=z(T, \cdot)=0$ in $\Omega$, and

$$
\int_{Q} \Pi_{1} q^{2} \mathrm{~d} x \mathrm{~d} t=-\int_{Q} f \cdot \nabla z \mathrm{~d} x \mathrm{~d} t+\int_{\omega_{T}} \Pi_{1} q p \mathrm{~d} x \mathrm{~d} t
$$

Thanks to (6.7) we have

$a(p, p)=b_{q}(p) \leq C^{\frac{1}{2}}(Q, \omega, c)\left(\int_{Q} \Pi_{1}|q|^{2}\right)^{\frac{1}{2}}|a(p, p)|^{\frac{1}{2}}$,

and so

$$
a(p, p)=\int_{Q}\left(\Pi_{2}\right)^{-1} z^{2}+\int_{\omega_{T}}\left(\Pi_{1}\right)^{-1} u^{2} \leq C(Q, \omega, c) \int_{Q} \Pi_{1}|q|^{2} .
$$

Let us prove the following estimate:

$$
\int_{Q}\left(\Pi_{2}\right)^{-1} z^{2}+\int_{\omega_{T}}\left(\Pi_{1}\right)^{-1} u^{2}+\int_{Q}\left(\Pi_{3}\right)^{-1}|\nabla z|^{2} \leq C(Q, \omega, c) \int_{Q} \Pi_{1} q^{2} .
$$


We set

$$
\begin{aligned}
I_{1} & :=\int_{Q}\left(\Pi_{3}\right)^{-1} c|\nabla z|^{2}, \\
I_{2} & :=-\int_{Q}\left(\Pi_{3}\right)^{-1} z \operatorname{div}(c \nabla z) \mathrm{d} x \mathrm{~d} t, \\
I_{3} & :=-2 \int_{Q} \nabla\left(\Pi_{3}^{-1}\right) z c \nabla \beta \cdot \nabla z \mathrm{~d} x \mathrm{~d} t, \\
I_{4} & :=\int_{Q}\left(\Pi_{3}\right)^{-1} z u \mathrm{~d} x \mathrm{~d} t \\
I_{5} & :=\int_{Q}\left(\Pi_{3}\right)^{-1} \Pi_{1} z q \mathrm{~d} x \mathrm{~d} t, \\
I_{6} & :=\int_{Q}\left(\Pi_{3}\right)^{-1} z \partial_{t} z \mathrm{~d} x \mathrm{~d} t .
\end{aligned}
$$

The Green formula holds in $Q$ with the weight $\mathrm{d} x \mathrm{~d} t$ for the product $\left(\Pi_{3}\right)^{-1} z \operatorname{div}(c \nabla z)$, since $c \nabla \beta=c \frac{\partial \beta}{\partial n_{S}} n_{S}$ on $S$ and the jump $\left[c \frac{\partial z}{\partial n}\right]$ vanishes. Thus $I_{1}=I_{2}+I_{3}$. Moreover, since $-\operatorname{div}(c \nabla z)=\mathcal{L}^{*} z+\partial_{t} z=u+\Pi_{1} q+\partial_{t} z$, then $I_{2}=I_{4}+I_{5}+I_{6}$ and

$$
I_{1}=I_{3}+I_{4}+I_{5}+I_{6} \text {. }
$$

Let us estimate $I_{k}, 3 \leq k \leq 6$. This originate from

Lemma 6.1 We have the following estimates:

$$
\begin{aligned}
& \left.\Pi_{1} \Pi_{2} \leq C\left(\Pi_{3}\right)^{2} \quad \text { (or equivalently: }\left(\Pi_{3}\right)^{-1} \leq C\left(\Pi_{1} \Pi_{2}\right)^{-\frac{1}{2}}\right), \\
& \lambda^{-2}\left(\Pi_{1}\right)^{2} \Pi_{2} \leq C\left(\Pi_{3}\right)^{3} \\
& \left|\nabla \Pi_{3}\right| \leq C \lambda^{-1} \Pi_{1} \\
& \left|\partial_{t} \Pi_{3}\right| \leq C s^{-1} \lambda^{-2}\left(\Pi_{2}\right)^{-1}\left(\Pi_{3}\right)^{2},
\end{aligned}
$$

where $C=C(Q, \omega, c)$.

Proof - Estimates (6.13), (6.14) are obvious. We have $\nabla \Pi_{3}=2 s^{2} \lambda^{3} \varphi^{2}(1+s \varphi) \mathrm{e}^{-2 s \eta} \nabla \beta$. Thus (6.15) holds if $s$ and $\lambda$ are sufficiently large. Thanks to (2.1), we have

$$
\left|\partial_{t} \Pi_{3}\right|=\left|2 s^{2} \lambda^{2} \varphi\left(\partial_{t} \varphi-s \varphi \partial_{t} \eta\right) \mathrm{e}^{-2 s \eta}\right| \leq C s^{3} \lambda^{2} \varphi^{4} \mathrm{e}^{-2 s \eta} .
$$

Thus

$$
\Pi_{2}\left|\partial_{t} \Pi_{3}\right| \leq C s^{3} \lambda^{2} \varphi^{4} \mathrm{e}^{-4 s \eta} \leq C s^{-1} \lambda^{-2}\left(s^{4} \lambda^{4} \varphi^{4} \mathrm{e}^{-4 s \eta}\right)=C\left(\Pi_{3}\right)^{2} .
$$

Hence we obtain (6.16).

We have $\left|I_{3}\right| \leq C \int_{Q} \lambda^{-1} \Pi_{1}\left(\Pi_{3}\right)^{-2}|z||\nabla z|$ thanks to $(6.15)$, then

$$
\left|I_{3}\right| \leq \frac{1}{2} I_{1}+2\left(c_{\text {min }}\right)^{-1} C \int_{Q} \lambda^{-2}\left(\Pi_{1}\right)^{2}\left(\Pi_{3}\right)^{-3}|z|^{2} \leq \frac{1}{2} I_{1}+C \int_{Q}\left(\Pi_{2}\right)^{-1}|z|^{2},
$$

thanks to the Minkovski estimate and to (6.14). Thus we obtain

$$
\left|I_{3}\right| \leq \frac{1}{2} I_{1}+C \int_{Q} \Pi_{1}|q|^{2}
$$


Thanks to (6.13) and to the Minkovski estimate, we have

$$
\begin{aligned}
& \left|I_{4}\right| \leq C \int_{Q}\left(\Pi_{2}\right)^{-1} z^{2}+C \int_{Q}\left(\Pi_{1}\right)^{-1} u^{2} \leq C^{\prime} \int_{Q} \Pi_{1}|q|^{2}, \\
& \left|I_{5}\right| \leq C \int_{Q}\left(\Pi_{2}\right)^{-1} z^{2}+C \int_{Q} \Pi_{1} q^{2} \leq C^{\prime} \int_{Q} \Pi_{1}|q|^{2} .
\end{aligned}
$$

By using an integration by parts with respect to the time variable, we have:

$$
I_{6}=\frac{1}{2} \int_{Q}\left(\Pi_{3}\right)^{-1} \partial_{t}\left(z^{2}\right) \mathrm{d} x \mathrm{~d} t=-\frac{1}{2} \int_{Q} \partial_{t}\left(\Pi_{3}^{-1}\right) z^{2} \mathrm{~d} x \mathrm{~d} t,
$$

and so, thanks to $(6.16)$,

$$
\left|I_{6}\right| \leq C s^{-1} \lambda^{-2} \int_{Q}\left(\Pi_{2}\right)^{-1} z^{2} \leq C \int_{Q} \Pi_{1} q^{2} .
$$

From (6.12), (6.17), (6.18), (6.19), (6.20) and (6.12), we deduce that $\left|I_{1}\right| \leq C \int_{Q} \Pi_{1} q^{2}$, and hence (6.11).

Let us resume (6.3). We have

$$
\begin{aligned}
\int_{Q} \Pi_{1} q^{2} & =\int_{Q} q(g-u)=-\int_{Q} f \cdot \nabla z-\int_{\omega_{T}} q u \\
& \leq\left(\int_{Q}\left(\Pi_{3}\right)^{-1}|\nabla z|^{2}\right)^{\frac{1}{2}}\left(\int_{Q} \Pi_{3}|f|^{2}\right)^{\frac{1}{2}}+\left(\int_{\omega_{T}}\left(\Pi_{1}\right)^{-1} u^{2}\right)^{\frac{1}{2}}\left(\int_{\omega_{T}} \Pi_{1} q^{2}\right)^{\frac{1}{2}} \\
& \leq C^{\frac{1}{2}}(Q, \omega, c)\left(\int_{Q} \Pi_{1} q^{2}\right)^{\frac{1}{2}}\left(\left(\int_{Q} \Pi_{3}|f|^{2}\right)^{\frac{1}{2}}+\left(\int_{\omega_{T}} \Pi_{1} q^{2}\right)^{\frac{1}{2}}\right),
\end{aligned}
$$

and thus:

$$
\int_{Q} \Pi_{1} q^{2} \leq C(Q, \omega, c)\left(\int_{Q} \Pi_{3}|f|^{2}+\int_{\omega_{T}} \Pi_{1} q^{2}\right) .
$$

That is, (2.5) holds.

Proof for Case 2 Let us prove that (2.7) holds for case 2. We use again the above method for case 3 with the weights $\Pi_{k}, k \in[1,3]$, modified as follows. Instead of (6.4) we set

$\Pi_{1}(t, x):=\sum_{i=1,2} s^{3} \lambda^{4} \varphi_{i}^{3} \mathrm{e}^{-2 s \eta_{i}}, \Pi_{2}(t, x):=\sum_{i=1,2} \mathrm{e}^{-2 s \eta_{i}}, \Pi_{3}(t, x):=\sum_{i=1,2} s^{2} \lambda^{2} \varphi_{i}^{2} \mathrm{e}^{-2 s \eta_{i}}(.6 .22)$

Note that lemma 6.1 still holds with these modifications. In fact, if for instance $\beta_{1}(x) \geq \beta_{2}(x)$, then

$$
\Pi_{1}(t, x) \Pi_{2}(t, x) \leq 4 s^{3} \lambda^{4} \varphi_{1}^{3}(t, x) \mathrm{e}^{-4 s \eta_{1}(t, x)} \leq 4\left(\Pi_{3}(t, x)\right)^{2} .
$$

Proofs of estimates (6.13), (6.14), (6.15) and (6.16) are similarly completed. Thus estimate (6.11) still holds. Then we straightforwardly obtain (6.21) and (2.7). 
Proof for Case 1 Let us prove that (2.9) holds for case 1. We assume that $f \in$ $\left(L^{2}(Q)\right)^{n} \cap\left(L^{2}\left(t_{0}, T ; H^{1}\left(V_{\gamma}\right)\right)\right)^{n}$, and $q \in L^{2}(Q) \cap L^{2}\left(t_{0}, T ; H^{2}\left(V_{\gamma}\right)\right)$, where $V_{\gamma}$ denotes a neighbourhood of $\gamma$ in $\Omega$. As already shown in part 2.1, we extend $\Omega$ to a smooth domain $\hat{\Omega} \subset \mathbb{R}^{n}$ and we fix $\omega \subset \subset \hat{\Omega} \backslash \Omega$. We set $\hat{Q}=\left(t_{0}, T\right) \times \hat{\Omega}$. We extend $q$ (respectively $f$ ) by setting $q=0$ (respectively $f=0$ ) in $\hat{Q} \backslash Q$, and we extend $c_{0}$ smoothly in such a way that $0<c_{\min } \leq c_{0}(x) \leq c_{\max }<\infty$ in $\hat{\Omega}$. This is thus the situation of case 2 with $\hat{\Omega}$ replacing $\Omega$, and we define $\Pi_{k}, k=1,2,3$ in $\hat{Q}$ by (6.22) again. Note that we have now $\mathcal{L} q=(\operatorname{div} f) \chi_{Q}+\left(c_{0} \partial_{n} q\right) \delta_{\gamma_{T}}$ in $\hat{Q}$, that is, $q$ satisfies $(6.23)$ :

$\int_{\hat{Q}} q g \mathrm{~d} x \mathrm{~d} t=-\int_{\hat{Q}} f \cdot \nabla z \mathrm{~d} x \mathrm{~d} t+\int_{\gamma_{T}}\left(f \cdot n+c \partial_{n} q\right) z \mathrm{~d} \sigma(x) \mathrm{d} t+\int_{\hat{\Omega}} q\left(t_{0}\right) z\left(t_{0}\right) \mathrm{d} x$,

where $z \in D\left(\hat{L}^{*}\right)$ satisfies $\hat{L}^{*} z=g, z(T)=0$, and $\left(\hat{L}^{*}, D\left(\hat{L}^{*}\right)\right)$ is defined as $\left(L^{*}, D\left(L^{*}\right)\right)$ but with $\Omega$ replaced by $\hat{\Omega}$.

Thus by comparison to case 2 (with $\Omega$ replaced by $\hat{\Omega}$ ), calculations are modified by taking account additional terms that originate from $\left(c_{0} \partial_{n} q\right) \delta_{\gamma_{T}}$. Since $q=0$ in $\hat{\Omega} \backslash \Omega$, (6.9) is replaced by

$$
\int_{Q} \Pi_{1} q^{2}=-\int_{Q} f \cdot \nabla z+\int_{\gamma_{T}}\left(f \cdot n+c_{0} \partial_{n} q\right) z
$$

and (6.11) holds with $\hat{\Omega}$ instead of $\Omega$. Thus, what we only have to do is prove that, if we set $J:=\int_{\gamma_{T}}\left(f \cdot n+c_{0} \partial_{n} q\right) z$ and $\Pi_{5}(t, x):=\sum_{i=1,2} s \lambda \varphi_{i} \mathrm{e}^{-2 s \eta_{i}}$, then

$$
|J| \leq C\left(\int_{Q} \Pi_{1} q^{2}\right)^{\frac{1}{2}}\left(\int_{\gamma_{T}} \Pi_{5}\left|f \cdot n+c_{0} \partial_{n} q\right|^{2}\right)^{\frac{1}{2}} .
$$

Let us set $K:=\int_{\gamma_{T}}\left(\Pi_{5}\right)^{-1} z^{2}$. We prove that $K \leq C \int_{Q} \Pi_{1} q^{2}$, which implies (6.25), thanks to the Cauchy-Schwarz estimate. Let us estimate $K$. This originate from

Lemma 6.2 We have the following estimates:

$$
\begin{aligned}
& \Pi_{2} \leq C \Pi_{5}, \\
& \left.\Pi_{2} \Pi_{3} \leq C\left(\Pi_{5}\right)^{2} \quad \text { or equivalently: }\left(\Pi_{5}\right)^{-1} \leq C\left(\Pi_{2} \Pi_{3}\right)^{-\frac{1}{2}}\right), \\
& \left|\nabla \Pi_{5}\right| \leq C \Pi_{3}, \\
& \left|\nabla\left(\Pi_{5}\right)^{-1}\right| \leq C\left(\Pi_{2}\right)^{-1},
\end{aligned}
$$

where $C=C(Q, \omega, c)$.

Proof - Estimates (6.26) and (6.27) are proved as in lemma 6.1 adapted for case 2. We have $\nabla \Pi_{5}=\sum_{i=1,2} s \lambda^{2} \varphi_{i}\left(1+2 s \varphi_{i}\right) \mathrm{e}^{-2 s \eta_{i}} \nabla \beta_{i}$. Then (6.28) holds.

Since $\nabla\left(\Pi_{5}\right)^{-1}=-\left(\Pi_{5}\right)^{-2} \nabla\left(\Pi_{5}\right)$, then, thanks to (6.28) and $(6.27)$, we have $\left|\nabla\left(\Pi_{5}\right)^{-1}\right| \leq$ $C\left(\Pi_{5}\right)^{-2} \Pi_{3} \leq C^{\prime}\left(\Pi_{2}\right)^{-1}$.

Note that for any $w \in W^{1,1}(\Omega)$ we have

$$
\int_{\gamma} w(x) \mathrm{d} \sigma(x) \leq C(\Omega, \gamma) \int_{\Omega}(|w|+|\nabla w|) \mathrm{d} x .
$$


We set $w=\left(\Pi_{5}\right)^{-1} z^{2}$. Thanks to (6.30) and lemma 6.2, we have

$$
\begin{aligned}
K=\int_{\gamma_{T}} w & \leq C(\Omega, \gamma) \int_{Q} \sum_{j=1,2}\left(\Pi_{5}^{-1}+\left|\nabla\left(\Pi_{5}^{-1}\right)\right|\right) z^{2}+2 \Pi_{5}^{-1}|z||\nabla z| \\
& \leq C(Q, \gamma, c) \int_{Q} \Pi_{2}^{-1} z^{2}+\Pi_{2}^{-\frac{1}{2}}|z| \Pi_{3}^{-\frac{1}{2}}|\nabla z| .
\end{aligned}
$$

Thanks to the Minskovski estimate and to (6.11), we obtain $K \leq C \int_{Q} \Pi_{1} q^{2}$. Hence (2.9) is proved.

\subsection{Proof of Lemma 3.1}

Let $b_{j} \in \mathcal{C}^{1}(\bar{\Omega})$ be an extension of $b_{\mid \Omega_{j}}$ to $\Omega_{1-j}, j=0,1$. Let $\varepsilon>0$ be arbitrary small. Since $\mathcal{C}^{2 n^{\prime}+2}(\bar{\Omega})$ is dense in $\mathcal{C}^{1}(\bar{\Omega})$, there exist $b_{j}^{\varepsilon} \in \mathcal{C}^{2 n^{\prime}+2}(\bar{\Omega}), j=0,1$, such that

$$
\left\|b_{j}^{\varepsilon}-b_{j}\right\|_{W^{1, \infty}(\Omega)} \leq \varepsilon .
$$

Now we "glue" $b_{0}^{\varepsilon}$ and $b_{1}^{\varepsilon}$ on $S$ in aim to obtain two condition transmissions. More precisely we prove that there exist an neighbourhood $U \subset \subset \Omega$ of $S$ which does not depend on $\varepsilon$ and a function $\mu_{1}^{\varepsilon} \in \mathcal{C}^{2 n^{\prime}+2}(\bar{\Omega})$ such that $\mu_{1}^{\varepsilon}=0$ in $\bar{\Omega} \backslash U, \mu_{1}^{\varepsilon}=b_{1}^{\varepsilon}-b_{0}^{\varepsilon}$ on $S, \partial_{n} \mu_{1}^{\varepsilon}=g^{\varepsilon} \equiv \frac{c_{1}}{c_{0}} \partial_{n} b_{1}^{\varepsilon}-\partial_{n} b_{0}^{\varepsilon}$ on $S$, and $\mu_{1}^{\varepsilon} \rightarrow 0$ in $W^{1, \infty}(\Omega)$ as $\varepsilon$ goes to 0 .

Since $S$ is compact and of class $\mathcal{C}^{2 n^{\prime}+2}$, there exists an open neighbourhood $W$ of $S$ in $\Omega$ of the form

$$
W=\left\{x=X\left(z^{\prime}, z_{n}\right) ; z^{\prime} \in S \text { and }-\varepsilon_{S}<z_{n}<\varepsilon_{S}\right\}
$$

where $X\left(\cdot, z_{n}\right)$ is a flow of class $\mathcal{C}^{2 n^{\prime}+2}(S)$ such that $X\left(z^{\prime}, 0\right)=z^{\prime}, X\left(z^{\prime}, z_{n}\right) \in \Omega_{0}$ if $0<z_{n}<\varepsilon_{S}$ and $X\left(z^{\prime}, z_{n}\right) \in \Omega_{1}$ if $0<-z_{n}<\varepsilon_{S}$, for all $z^{\prime} \in S$ and some $\varepsilon_{S}>0$.

Moreover we have $\frac{\partial}{\partial z_{n}}=\partial_{n}$ on $S$. For a proof of this, we refer to [15]. To simplify we identify $z$ and $x$. We define on $W$ the mappings $Z^{\prime}$ and $Z_{n}$ of class $\mathcal{C}^{2 n^{\prime}+2}$ by $Z^{\prime}(x):=z^{\prime}$ and $Z_{n}(x)=z_{n}$. We set

$$
\begin{aligned}
& \alpha_{0}:=\sqrt{\varepsilon}, \\
& \alpha_{1}:=\varepsilon\left(1+\left\|g^{\varepsilon}\right\|_{W^{1, \infty}(S)}\right)^{-1} .
\end{aligned}
$$

Notice that $\alpha_{j}$ tends to 0 as $\varepsilon$ goes to $0, j=0,1$. Let $\psi \in \mathcal{C}_{0}^{\infty}(\mathbb{R})$ be such that $\psi(r)=1$ if $|r| \leq \frac{1}{2}, \psi(r)=0$ if $|r| \geq 1$. We set $a_{n}:=\left(\int_{\mathbb{R}^{n}} \psi(|\zeta|) d^{n} \zeta\right)^{-1}, \psi_{j}^{\varepsilon}(x)=\psi\left(z_{n} / \alpha_{j}\right)$, $j=0,1$, and

$\mu_{1}^{\varepsilon}(x):=\psi_{0}^{\varepsilon}(x)\left(b_{1}^{\varepsilon}\left(z^{\prime}\right)-b_{0}^{\varepsilon}\left(z^{\prime}\right)\right)+\psi_{1}^{\varepsilon}(x) z_{n} \int_{\zeta \in \mathbb{R}^{n}} a_{n} \psi(|\zeta|) g^{\varepsilon}\left(Z^{\prime}\left(x-z_{n} \zeta\right)\right) \mathrm{d}^{n} \zeta$.

Notice that $\left|Z_{n}\left(x-z_{n} \zeta\right)\right| \leq C_{S}\left|z_{n}\right|$ if $|\zeta| \leq 1$ where $C_{S} \geq 1$ depends only on $S$. Hence $\mu_{1}^{\varepsilon}(x)$ is well defined if $\left|z_{n}\right| \leq \varepsilon_{S}^{\prime} \equiv\left(C_{S}\right)^{-1} \varepsilon_{S} \equiv \varepsilon_{S}^{\prime}$. If $x \notin W$ or if there exists $\zeta \in \mathbb{R}^{n}$ with $|\zeta| \leq 1$ and $\left|Z_{n}\left(x-z_{n} \zeta\right)\right| \geq \varepsilon_{S}$ then we set $\mu_{1}^{\varepsilon}(x)=0$.

We have $\mu_{1 \mid S}^{\varepsilon}=\left(b_{1}^{\varepsilon}-b_{0}^{\varepsilon}\right)_{\mid S}$ and we can easily check that $\mu_{1}^{\varepsilon} \rightarrow 0$ as $\varepsilon$ goes to 0 , uniformly in $\Omega$, since from (6.31) and the fact that $[b]=0$ on $S$ we obtain $\left|\left(b_{1}^{\varepsilon}-b_{0}^{\varepsilon}\right)_{\mid S}\right| \leq 2 \varepsilon$.

For all $x \in W$ with $\left|z_{n}\right| \leq \varepsilon_{S}^{\prime}$ we compute:

$\partial_{z_{n}} \mu_{1}^{\varepsilon}=\psi^{\prime}\left(\frac{z_{n}}{\alpha_{0}}\right)\left(b_{1}^{\varepsilon}\left(z^{\prime}\right)-b_{0}^{\varepsilon}\left(z^{\prime}\right)\right) / \varepsilon^{\frac{1}{2}}+$ 


$$
\begin{aligned}
& \left(\left(\frac{z_{n}}{\alpha_{1}}\right) \psi^{\prime}\left(\frac{z_{n}}{\alpha_{1}}\right)+\psi\left(\frac{z_{n}}{\alpha_{1}}\right)\right) \cdot \int_{\zeta \in \mathbb{R}^{n}} a_{n} \psi(|\zeta|) g^{\varepsilon}\left(Z^{\prime}\left(x-z_{n} \zeta\right)\right) \mathrm{d}^{n} \zeta \\
& +\frac{z_{n}}{\alpha_{1}} \psi\left(\frac{z_{n}}{\alpha_{1}}\right) \int_{\zeta \in \mathbb{R}^{n}} a_{n} \psi(|\zeta|)\left(\frac{\partial X}{\partial z_{n}}-\zeta\right) \cdot \alpha_{1} \nabla\left(g^{\varepsilon} \circ Z^{\prime}\right)\left(x-z_{n} \zeta\right) \mathrm{d}^{n} \zeta .
\end{aligned}
$$

Thanks to the characteristics of the convolution, we can compute:

$$
\begin{aligned}
\partial_{z^{\prime}} \mu_{1}^{\varepsilon}= & \psi_{0}^{\varepsilon}(x) \partial_{z^{\prime}}\left(b_{1}^{\varepsilon}\left(z^{\prime}\right)-b_{0}^{\varepsilon}\left(z^{\prime}\right)\right) \\
& +\psi_{1}^{\varepsilon}(x)\left|z_{n}\right| \int_{\zeta \in \mathbb{R}^{n}} \frac{\partial X}{\partial z^{\prime}} \cdot \frac{\zeta}{|\zeta|} a_{n} \psi^{\prime}(|\zeta|) g^{\varepsilon}\left(Z^{\prime}\left(x-z_{n} \zeta\right)\right) \mathrm{d}^{n} \zeta .
\end{aligned}
$$

We can check that $\left(\partial_{z_{n}} \mu_{1}^{\varepsilon}\right)\left(X\left(z^{\prime}, 0\right)\right)=g^{\varepsilon}\left(z^{\prime}\right)$. That is to say, $\left(\partial_{n} \mu_{1}^{\varepsilon}\right)_{\mid S}=g^{\varepsilon}$. Moreover from (6.36) we obtain that $\partial_{z^{\prime}} \mu_{1}^{\varepsilon}(x)$ is bounded as $\varepsilon \rightarrow 0$, uniformly to $x \in \bar{\Omega}$. From (6.35) we obtain that $\partial_{z_{n}} \mu_{1}^{\varepsilon}(x) \rightarrow 0$ as $\varepsilon \rightarrow 0$, uniformly to $x \in \bar{\Omega}$, thanks to $(6.31),(6.32),(6.33)$. Moreover, since $\left[c \partial_{n} b\right]=0$ on $S$, then $g^{\varepsilon}$ tends to 0 in $L^{\infty}(S)$. Thus $\mu_{1}^{\varepsilon} \rightarrow 0$ in $W^{1, \infty}(\Omega)$.

We set $b_{2}^{\varepsilon}=b_{1}^{\varepsilon} \chi_{\overline{\Omega_{1}}}+\chi_{\Omega_{0}}\left(\mu_{1}^{\varepsilon}+b_{0}^{\varepsilon}\right)$. Thus we have $b_{2 \mid \Omega_{j}}^{\varepsilon} \in \mathcal{C}^{2 n^{\prime}+2}\left(\overline{\Omega_{j}}\right), j=0,1$, and

$$
\begin{aligned}
& {\left[b_{2}^{\varepsilon}\right]=0 \text { and }\left[c \partial_{n} b_{2}^{\varepsilon}\right]=0 \text { on } S ;} \\
& b_{2}^{\varepsilon} \rightarrow b \text { as } \varepsilon \text { goes to } 0, \text { in } W^{1, \infty}(\Omega) .
\end{aligned}
$$

We construct now a function $\mu_{2}^{\varepsilon}$ satisfying

$$
\begin{aligned}
& \mu_{2 \mid \Omega_{1}}^{\varepsilon}=0 \text { and } \mu_{2 \mid \Omega_{0}}^{\varepsilon} \in \mathcal{C}^{2 n^{\prime}+2}\left(\overline{\Omega_{0}}\right), \\
& {\left[\mu_{2}^{\varepsilon}\right]=0 \text { and }\left[c \partial_{n} \mu_{2}^{\varepsilon}\right]=0 \text { on } S ;} \\
& {\left[\mathcal{A}^{l} \mu_{2}^{\varepsilon}\right]=-\left[\mathcal{A}^{l} b_{2}^{\varepsilon}\right] \text { and }\left[c \partial_{n} \mathcal{A}^{l} \mu_{2}^{\varepsilon}\right]=-\left[c \partial_{n} \mathcal{A}^{l} b_{2}^{\varepsilon}\right] \text { on } S, l=1, \ldots, n^{\prime} .}
\end{aligned}
$$

In the change of variable $x \mapsto z=Z(x)$, the mapping $q \rightarrow \mathcal{A}_{0} q$ can be written as $w \rightarrow B w$ where $w=q \circ X$ and $B$ is a two order linear mapping of the form $-\left(c_{0} \circ X\right) \partial_{z_{n}}^{2}+\partial_{z_{n}} B_{1}+B_{2}$ where $B_{i}$ are partial differential operators on the variable $z^{\prime}$ of order $i$ with coefficients in $\mathcal{C}^{2 n^{\prime}}\left(W_{0}\right), i=1,2$, where $W_{0} \equiv \overline{\Omega_{0}} \cap W$. Thus, thanks to $(6.37)$, conditions $(6.39)$ can be written as

$$
\begin{aligned}
& \left(\partial_{z_{n}}^{2 l} \mu_{2}^{\varepsilon}\right)_{\mid S}=F_{2 l}\left(\left[\mathcal{A}^{l} b_{2}^{\varepsilon}\right],\left(\partial_{z_{n}}^{j} \partial_{z^{\prime}}^{k} \mu_{2}^{\varepsilon}\right)_{\mid S} ; j=0, \ldots, 2 l-1 ; k=0, \ldots, 2 l-j\right) \\
& \left(\partial_{z_{n}}^{2 l+1} \mu_{2}^{\varepsilon}\right)_{\mid S}=F_{2 l+1}\left(\left[c \partial_{n} \mathcal{A}^{l} b_{2}^{\varepsilon}\right],\left(\partial_{z_{n}}^{j} \partial_{z^{\prime}}^{k} \mu_{2}^{\varepsilon}\right)_{\mid S} ; j=0, \ldots, 2 l ; k=0, \ldots, 2 l+1-j\right),
\end{aligned}
$$

where $F_{j}$ are linear mappings with coefficients in $\mathcal{C}^{2 n^{\prime}+2-j}(S), j=2, \ldots, 2 n^{\prime}+1$. Moreover, notice that $\left[\mathcal{A}^{l} b_{2}^{\varepsilon}\right] \in \mathcal{C}^{2 n^{\prime}+2-2 l}(S)$ and $\left[c \partial_{n} \mathcal{A}^{l} b_{2}^{\varepsilon}\right] \in \mathcal{C}^{2 n^{\prime}+1-2 l}(S), l \leq n^{\prime}$. Thus we built $\mu_{2}^{\varepsilon} \in \mathcal{C}^{2 n^{\prime}+2}\left(W_{0}\right)$ such that (6.40) and (6.41) hold by an iterative process on $l \in\left[1, n^{\prime}\right]$. Indeed we fix $\mu_{2}^{\varepsilon}=0$ on $S$ and $\partial_{z_{n}} \mu_{2}^{\varepsilon}=0$ on $S$ to start the process. Then we apply [?, Volume I, Corollary 1.3.4] which says that given arbitrary $u_{j} \in \mathcal{C}^{k-j}\left(\mathbb{R}^{n}\right)$, $0 \leq j \leq k$, on can find $u \in \mathcal{C}^{k}\left(\mathbb{R}^{n+1}\right)$ so that $\partial_{t}^{j} u(x, t)=u_{j}(x)$ when $t=0, j=0, \ldots, k$. Moreover, thanks to (6.37) and (6.38) the function $h^{\varepsilon}:=\left(\left(z_{n}\right)^{-2} \mu_{2}^{\varepsilon}\right)_{\mid W_{0}}$ is continuously differentiable in $W_{0}$. We set

$$
\alpha_{2}=\varepsilon\left(1+\left\|h^{\varepsilon}\right\|_{W^{1, \infty}\left(W_{0}\right)}\right)^{-1}>0,
$$

and $\mu_{3}^{\varepsilon}(x)=\psi\left(z_{n} / \alpha_{2}\right) \mu_{2}^{\varepsilon}(x)$ for $x \in W, \mu_{3}^{\varepsilon}(x)=0$ for $x \notin W$. Then $\mu_{3}^{\varepsilon}$ satisfies (6.37), (6.38), (6.39). Notice that both $\alpha_{2}$ and $\alpha_{2}\left\|h^{\varepsilon}\right\|_{W^{1, \infty}\left(W_{0}\right)}$ tend to 0 as $\varepsilon$ goes to 0 . Hence $\mu_{3}^{\varepsilon}$ satisfies the following property:

$$
\mu_{3}^{\varepsilon} \rightarrow 0 \text { as } \varepsilon \text { goes to } 0 \text {, in } W^{1, \infty}(\Omega) \text {. }
$$


In addition, since $\Gamma$ is of class $\mathcal{C}^{2 n^{\prime}+2}$ (as $S$ ), the above method shows that we can choose $b_{1}^{\varepsilon}$ satisfying $\mathcal{A}_{1}^{l} b_{1}^{\varepsilon}=0$ on $\Gamma$ and $\partial_{n} \mathcal{A}_{1}^{l} b_{1}^{\varepsilon}=0$ on $\Gamma, l=1, \ldots, n^{\prime}$. Now we set

$$
b^{\varepsilon}=b_{2}^{\varepsilon}+\chi_{\Omega_{0}} \mu_{3}^{\varepsilon} .
$$

Then, thanks to proposition 3.1, we obtain $b^{\varepsilon} \in D\left(A^{n^{\prime}+1}\right)$. Finally, thanks to the characteristics of $b_{2}^{\varepsilon}$ and $\mu_{3}^{\varepsilon}$ we have $b^{\varepsilon} \rightarrow b$ in $W^{1, \infty}(\Omega)$ as $\varepsilon$ goes to 0 .

End of proof.

\subsection{Proof of Proposition 3.4}

We give a formal proof. To be rigourous, we could use the spectral representation of operator $A$.

We know that if $H \in L^{2}\left(Q_{0}\right)$ then $q \in \mathcal{C}^{0}\left([0, T], D\left(A^{\frac{1}{2}}\right)\right)$. (Notice that $\left.D\left(A^{\frac{1}{2}}\right)=H_{0}^{1}(\Omega)\right)$. Indeed we have

$$
q(t, \cdot)=Y\left(0, q_{0}\right)(t, \cdot)+\int_{0}^{t} e^{(s-t) A} H(s, \cdot) d s .
$$

Remark 6.2 $Y\left(0, q_{0}\right)(t, \cdot)=e^{-t A} q_{0}$ is defined for all $q_{0} \in D\left(A^{-m}\right)$, $m$ fixed in $\mathbb{N}$, and $\left.\left.Y\left(0, q_{0}\right) \in \mathcal{C}^{0}\left([0, T], D\left(A^{-m}\right)\right) \cap \mathcal{C}^{0}(] 0, T\right], D\left(A^{r}\right)\right)$ for all $r \in \mathbb{N}$.

We assume that $H \in H^{2}\left(0, T ; D\left(A^{k}\right)\right)$ for some $k \in \mathbb{N}$. We set $q^{k}:=A^{k} q$, $H^{k}:=A^{k} H \in H^{2}\left(0, T ; L^{2}(\Omega)\right), q_{0}^{k}:=A^{k} q_{0} \in D\left(A^{-k}\right)$. Then, thanks to remark 6.2, we have

$$
\left.\left.q^{k}=Y\left(H^{k}, q_{0}^{k}\right) \in \mathcal{C}^{0}\left([0, T], D\left(A^{-k}\right)\right) \cap \mathcal{C}^{0}(] 0, T\right], D\left(A^{\frac{1}{2}}\right)\right) .
$$

Since $\partial_{t} q^{k}=-A q^{k}+H^{k} \in \mathcal{C}^{0}\left([0, T], D\left(A^{-k-1}\right)\right)$ then $q_{1}^{k}:=\partial_{t} q^{k}(0, \cdot) \in D\left(A^{-k-1}\right)$. Since $\partial_{t} H^{k} \in H^{1}\left(0, T ; L^{2}(\Omega)\right)$, we then have

$$
\left.\left.\partial_{t} q^{k}=Y\left(\partial_{t} H^{k}, q_{1}^{k}\right) \in \mathcal{C}^{0}\left([0, T], D\left(A^{-k-1}\right)\right) \cap \mathcal{C}^{0}(] 0, T\right], D\left(A^{\frac{1}{2}}\right)\right) .
$$

Since $\partial_{t}^{2} q^{k}=-\partial_{t} A q^{k}+\partial_{t} H^{k} \in \mathcal{C}^{0}\left([0, T], D\left(A^{-k-2}\right)\right)$ then $q_{2}^{k}:=\partial_{t}^{2} q^{k}(0, \cdot) \in D\left(A^{-k-2}\right)$. Since $\partial_{t}^{2} H^{k} \in L^{2}\left(Q_{0}\right)$, we then have

$$
\left.\left.\partial_{t}^{2} q^{k}=Y\left(\partial_{t}^{2} H^{k}, q_{2}^{k}\right) \in \mathcal{C}^{0}\left([0, T], D\left(A^{-k-2}\right)\right) \cap \mathcal{C}^{0}(] 0, T\right], D\left(A^{\frac{1}{2}}\right)\right) .
$$

Hence, from (6.43), (6.44), (6.45) we obtain the conclusion.

\section{Acknowledgments}

The author wish to thanks A. Benabdallah, P. Gaitan, J. Le Rousseau and Y. Dermenjian for discussions on the subject of this article.

\section{References}

[1] P. Grisvard. Elliptic problems in nonsmooth domains. Pitman, Boston, London, Melbourne, 1985.

[2] A. Pazy. Semigroups of Linear Operators and Applications to Partial Differential Equations, volume 44. A.M.S, Springer-Verlag, New York, 1984. 
[3] A.L. Bukhgeim and M.V. Klibanov. Global uniqueness of a class of multidimensional inverse problems. Soviet Math. Dokl., 24:244-247, 1981.

[4] M.V. Klibanov. Global Uniqueness of a multidimensional Inverse Problem for a non linear parabolic equation by a Carleman Estimate. Inverse Problems, 20:1003-1032, 2004.

[5] O.Y. Imanuvilov and M. Yamamoto. Lipschitz stability in inverse problems by Carleman estimate. Inverse Problems, 14:1229-1245, 1998.

[6] L. Baudoin and J-P. Puel. Uniqueness and stability in an inverse problem for the Schrödinger equation. Inverse Problems, 18:1537-1554, 2002.

[7] V. Isakov. Carleman type estimates in an anisotropic case and applications. J. Diff. Eq., 105:217$238,1993$.

[8] A. Fursikov and O. Yu. Imanuvilov. Controllability of evolution equations. Seoul National University, Korea. Lecture Notes, 34, 1996.

[9] E. Fernàndez-Cara and S. Guerrero. Global Carleman inequalities for parabolic systems and application to Controllability. SIAM J. Control Optim, 45(4):1395-1446, 2006.

[10] H. Egger, H.W. Engl, and M.V. Klibanov. Global uniqueness and Hölder stability for recovering a non linear source term in a parabolic equation. Inverse Problems, 21:271-290, 2005.

[11] M.V. Klibanov and A. Timonov. Carleman Estimates For Coefficient Inverse Problems And Numerical Applications. Brill Academic Publishers, VSP, Utrecht, Boston, 2004.

[12] O.Y. Imanuvilov and M. Yamamoto. Determination of a coefficient in an acoustic equation with a single measurement. Inverse Problems, 19(2):157-171, 2003.

[13] M.V. Klibanov and M. Yamamoto. Lipschitz stability for an inverse problem for a acoustic equation. Applicable Analysis, 85(5):515-538, 2006.

[14] M. Yamamoto and G. Yuan. Lipschitz stability in the determination of principal parts of a parabolic equation by boundary observations. Technical Report UTMS 2005-36, University of Tokyo, 2005.

[15] A. Benabdallah, P. Gaitan, and J. Le Rousseau. Stability of discontinuous diffusion coefficients and initial conditions in an inverse problem for the heat equation. SIAM J. Control Optim (SICON), 2007.

[16] M.V. Klibanov. Inverse Problems and Carleman Estimates. Inverse Problems, 8:575-596, 1992.

[17] A. Doubova, A. Osses, and J.-P. Puel. Exact controllability to trajectories for semilinear heat equations with discontinuous diffusion coefficients. ESAIM COCV, 8:621-661, June 2002.

[18] O.Y. Imanuvilov and M. Yamamoto. Carleman Inequalities for Parabolic Equations in Sobolev Spaces of Negative Order and Exact Controllability for Semilinear Parabolic Equations. RIMS, Kyoto Univ., 39(2):227-274, 2003.

[19] O.Yu. Imanuvilov and M. Yamamoto. Carleman estimates for the non-stationary Lamé system and the application to an inverse problem. ESAIM COCV, 11:1-56, January 2005.

[20] W. P. Ziemer. Weakly differentiable functions. Springer-Verlag, Berlin, Heidelberg, New-York, Tokyo, 1989.

[21] A. Benabdallah, P. Gaitan, and J. Le Rousseau. Stability of discontinuous diffusion coefficients and initial conditions in an inverse problem for the heat equation. Technical report, LATP, Université de Provence, Marseille (web site: www.cmi.univ-mrs.fr/ lerous/publications.html), 2005.

[22] J. L. Lions and E. Magenes. Problèmes aux limites non homogènes et applications, volume 1. Dunod, Paris, 1968.

[23] Lars Hormander. Linear partial differential operators. Springer-Verlag, Berlin, 1963. 